This document is confidential and is proprietary to the American Chemical Society and its authors. Do not copy or disclose without written permission. If you have received this item in error, notify the sender and delete all copies.

\title{
Phase Behavior of Binary Mixture Systems of Saturated- Unsaturated Mixed-Acid Triacylglycerols: Effects of Glycerol Structures and Chain-Chain Interactions
}

\begin{tabular}{|r|l|}
\hline Journal: & The Journal of Physical Chemistry \\
\hline Manuscript ID: & jp-2015-00673j.R1 \\
\hline Manuscript Type: & Article \\
\hline Date Submitted by the Author: & n/a \\
\hline Complete List of Authors: & $\begin{array}{l}\text { Bayés-García, Laura; Universitat de Barcelona, Cristal·lografia, Mineralogia } \\
\text { i Dipòsits Minerals } \\
\text { Calvet, Teresa; Universitat de Barcelona, Cristal-lografia, Mineralogia i } \\
\text { Dipòsits Minerals } \\
\text { Cuevas-Diarte, Miquel; Universitat de Barcelona, Cristal·lografia, } \\
\text { Mineralogia i Dipòsits Minerals } \\
\text { Ueno, Satoru; Hiroshima University, Faculty of Applied Biological Science } \\
\text { Sato, Kiyotaka; Hiroshima University, Faculty of Applied Biological Science }\end{array}$ \\
\hline
\end{tabular}




\title{
Phase Behavior of Binary Mixture Systems of
}

\section{Saturated-Unsaturated Mixed-Acid Triacylglycerols:}

\section{Effects of Glycerol Structures and Chain-Chain}

\section{Interactions}

Laura Bayés-García, ${ }^{* a}$ Teresa Calvet ${ }^{a}$, Miquel Àngel Cuevas-Diarte ${ }^{a}$, Satoru Ueno ${ }^{b}$, and

Kiyotaka Sato ${ }^{b}$

\author{
${ }^{a}$ Departament de Cristal·lografia, Mineralogia i Dipòsits Minerals, Facultat de Geologia, \\ Universitat de Barcelona, Martí i Franquès s/n, E-08028 Barcelona, Spain. \\ ${ }^{b}$ Faculty of Applied Biological Science, Hiroshima University, Higashi-Hiroshima 739, Japan.
}




\begin{abstract}
We systematically examined the phase behavior of binary mixtures of mixed-acid triacylglycerols (TAGs) containing palmitic and oleic acid moieties, OPO (1, 3-dioleoyl-2palmitoyl-glycerol), PPO (1, 2-dipalmitoyl-3-oleoyl-rac-glycerol), and OOP (1,2-dioleoyl-3palmitoyl-rac-glycerol), which are widely present in natural fats and are employed in the food, pharmaceutical, and cosmetic industries. DSC and X-ray diffraction (XRD) methods were applied to observe the mixing behavior of PPO-OPO, OOP-OPO and PPO-OOP under metastable and stable conditions. The results led to three conclusions. (1) Eutectic behavior was observed in PPO-OPO. (2) Molecular-compound (MC) crystals were formed in the mixtures of OOP-OPO and PPO-OOP. (3) However, the MC crystals occurred only under metastable conditions and tended to separate into component-TAGs to form eutectic mixture systems after 17 months of incubation. These results were contrary to those of previous studies on POP-OPO and POP-PPO, in which the MC crystals were thermodynamically stable. We determined that specific molecular interactions may cause this different phase behavior (stability of POP:OPO and POP:PPO MC crystals, and metastability of OOP:OPO and PPO:OOP MC crystals). All results confirm the significant effects of molecular structures of glycerol groups, interactions of fatty acid chains, and polymorphism of the component TAGs on the mixing behavior of mixedacid TAGs.
\end{abstract}




\section{INTRODUCTION}

Lipids are major nutrients employed as lipophilic materials in the food, pharmaceutical, and cosmetic industries. In this large group of compounds, the crystals of triacylglycerols (TAGs) are main components of solid lipids employed in spreads, cream, and confections. ${ }^{1,2}$ The physicochemical properties of TAG crystals (e.g., melting, morphology, texture, and rheology) are determined mainly by their fatty acid compositions, polymorphism and mixing behavior of different TAGs as microscopic factors ${ }^{3}$ and by crystallization conditions under various external influences (e.g., cooling rates, shear, sonication, and additives) as macroscopic factors. ${ }^{4,5}$

The polymorphic behavior and the occurrence of multiple polymorphic forms of these lipid materials are strongly related to the chemical nature of the fatty acid components (chain length, saturated or unsaturated, and cis or trans double bonds). ${ }^{1}$ Mono-acid and mixed-acid TAGs are defined by whether three fatty acid moieties of the TAG are the same or not. Saturatedunsaturated mixed-acid TAGs are most commonly present in nature. For example, TAGs containing palmitic and oleic acid moieties are present in edible fats, ${ }^{6}$ vegetable fats and oils (palm oil ${ }^{7}$ and olive oil ${ }^{8}$ ), and animal fats. ${ }^{9,10}$ The polymorphic behavior of mixed-acid TAGS is more complicated than that of mono-acid TAGs, depending on the variation in fatty acid compositions of stereo-specific numbered $(s n)$ carbon atoms of the TAG. ${ }^{11,12}$ Specifically, molecular interactions of the aliphatic chains, methyl end stacking, and glycerol conformation of mixed-acid TAGs are modified compared to those of monoacid TAGs. As a consequence, the relative stability of multiple polymorphic forms and their melting/crystallization behavior are modified. ${ }^{1,13,14}$ 
Since most of the fats present in nature and employed in industry contain different types of mixed-acid TAGs, their physicochemical properties must be studied both in their pure systems and in mixed systems. For this purpose, it is necessary to examine mixing behavior in binary, ternary, and more complicated mixture systems. Three typical mixing states have been reported for the binary mixture systems of TAGs: (i) solid solution phase, (ii) eutectic phase, and (iii) molecular compound (MC) formation. A solid solution phase is formed when two component TAG molecules exhibit structural similarity and affinitive molecular interactions, so that the component molecules occupy the same crystallographic positions randomly. In the contrary case, when two component molecules are immiscible, eutectic equilibrium is obtained. ${ }^{15,16}$ A specific case is the formation of MC phase, which is formed only at clearly defined compositions through specific molecular interactions among the component TAG molecules. ${ }^{17}$

Recently, we systematically examined the binary-mixture systems of four major TAGs containing palmitic and oleic acid moieties of POP (1,3-dipalmitoyl-2-oleoyl glycerol), OOP (1,2-dioleoyl-3-palmitoyl-rac-glycerol), PPO (1,2-dipalmitoyl-3-oleoyl-rac-glycerol), and OPO (1,3-dioleoyl-2-palmitoyl-glycerol). Eutectic behavior was observed for the POP-OOP mixture, ${ }^{18}$ whereas MC formation at a ratio of 50:50 was observed in mixtures of POP-PPO ${ }^{19}$ and POPOPO. ${ }^{20} \mathrm{MC}$-forming mixtures of POP-OPO were observed in neat liquid and in solutions including $n$-dodecane as a solvent. ${ }^{21}$ The same properties were observed in the mixture systems of SOS (1,3-distearoyl-2-oleoyl glycerol), OOS (1,2-dioleoyl-3-stearoyl-rac-glycerol), SSO (1,2distearoyl-3-oleoyl-rac-glycerol), and OSO (1,3-dioleoyl-2-stearoyl-glycerol) as eutectic (SOS-

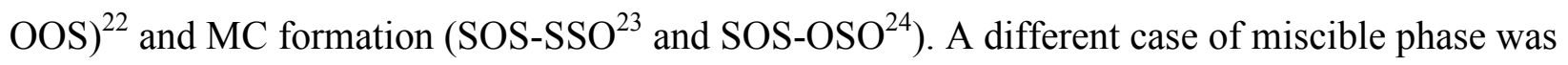
the mixture of SOS-SLS (1,3-distearoyl-2-linoleoyl glycerol). ${ }^{25}$ 
Having reviewed previous studies on mixture systems of POP-OOP, POP-PPO, and POPOPO, we determined that full understanding of the mixing behavior of symmetric and asymmetric mixed-acid TAGs must be obtained by systematically examining a tetragonal diagram of the binary mixture systems of POP, OPO, PPO, and OOP (Fig. 1).

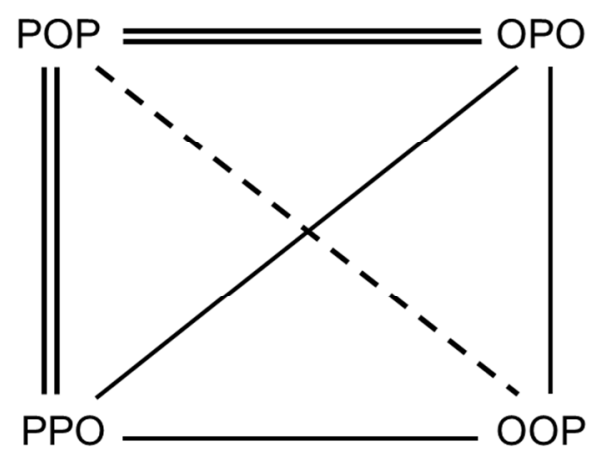

Molecular Compound (metastable and stable states) ${ }^{19,20}$

- - - - - Eutectic (metastable and stable states) ${ }^{18}$

Present study

Figure 1. Tetragonal diagram of binary mixture systems of POP, OPO, PPO and POO.

Figure 1 represents all possible binary mixtures among symmetric (POP and OPO) and asymmetric (PPO and OOP) TAGs. Clear contrasts are observed in the positions of sn-carbon atoms where the palmitic and oleic acid moieties are connected (e.g., POP vs OPO and PPO vs OOP). In addition, the most stable polymorphic forms differ among the four TAGs (e.g., $\beta$ form for $\mathrm{POP}^{26}$ and $\mathrm{OPO},,^{20,27}$ but $\beta^{\prime}$ form for $\mathrm{PPO}^{19}$ and $\left.\mathrm{OOP}^{28,29}\right)$. Therefore, it is quite interesting and important to determine how the molecular structures of the TAGs and their polymorphism can affect mixing behavior under metastable and most stable thermodynamic conditions. 
Table 1 lists the melting temperatures and the long and short spacings of the three TAGs that make up the binary mixtures studied in this work.

Table 1. Melting temperatures $\left(\mathrm{T}_{\mathrm{m}},{ }^{\circ} \mathrm{C}\right)$, long spacing (LS, nm) and short spacing (SS, nm) values of PPO, OPO and POO. Melting temperatures were defined by the peak top temperatures (data based on previous work $^{19,20,27,28,29}$ ).

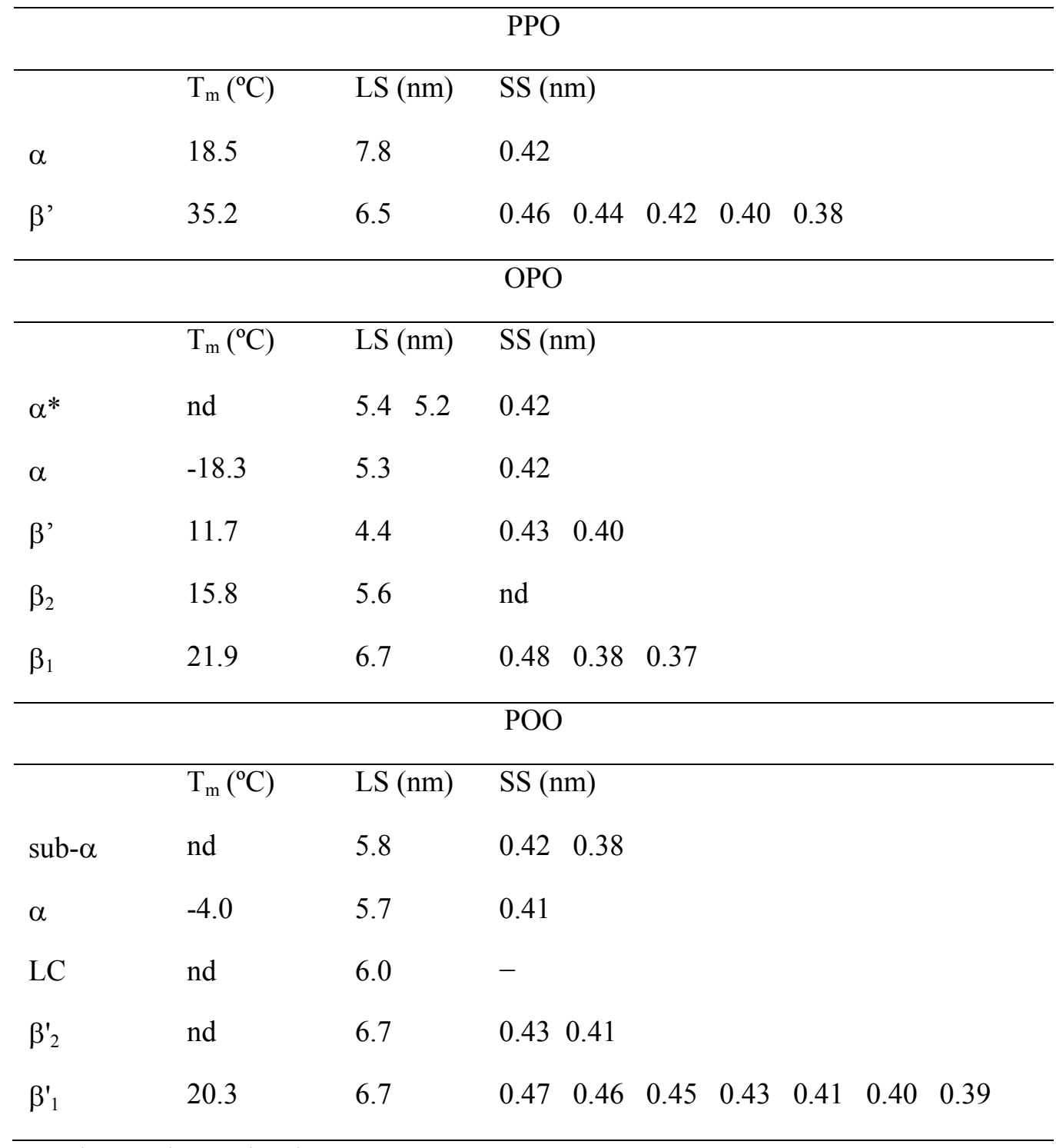

nd. Not determined 
Here, $\alpha$ and $\beta$ ' forms were determined in PPO, ${ }^{19}$ with $\alpha$ form having a long spacing of $7.8 \mathrm{~nm}$ and $\beta$ ' form having a long spacing of $6.5 \mathrm{~nm}$. More complicated polymorphism was observed in OPO, with five polymorphic forms already reported ${ }^{20,27}: \alpha^{*}$ (with long spacings of 5.4 and $5.2 \mathrm{~nm}), \alpha(5.3 \mathrm{~nm}), \beta^{\prime}(4.4 \mathrm{~nm}), \beta_{2}(5.6 \mathrm{~nm})$, and $\beta_{1}(6.7 \mathrm{~nm})$. Also, five phases were detected in $\mathrm{OOP}^{28,29}$ : sub- $\alpha$ form (having a long spacing of $\left.5.8 \mathrm{~nm}\right), \alpha$ form $(5.7 \mathrm{~nm})$, a liquid crystal (LC) phase (6.0nm), and $\beta^{\prime}{ }_{2}$ and $\beta{ }_{1}$ forms (both with long spacing of $6.7 \mathrm{~nm}$ ).

In the present study, we performed experiments on the crystallization and thermodynamic equilibration of PPO-OPO, OOP-OPO and PPO-OOP mixtures and compared the results with those of previous studies. The present work confirmed eutectic behavior in PPO-OPO mixtures, and the formation of MCs in OOP-OPO and PPO-OOP mixtures. However, these MCs occurred only under metastable conditions, and the systems evolved to eutectic behavior after 17 months of incubation, due to separation from MC to separated eutectic mixtures. All these results are quite new findings that can be applied to other TAGs containing various saturated and unsaturated fatty acid moieties that are present in many natural and industrial fats.

\section{MATERIALS AND METHODS}

Samples of PPO, OPO, and OOP (purity $>99 \%$ ) were purchased from Tsukishima Foods Industry Co., Ltd. (Tokyo, Japan) and used without further purification. Samples of asymmetric PPO consisted of a racemic mixture of the enantiomeric compounds R-PPO and S-PPO, and OOP consisted of R-OOP and S-OOP. 
In order to determine the phase behavior of PPO-OPO, OOP-OPO and PPO-OOP systems, TAG mixtures (\% molar) were prepared for every $10 \%$, melted at $50^{\circ} \mathrm{C}$, and mixed using a vortex.

DSC experiments were conducted at atmospheric pressure using a Perkin Elmer Diamond. Samples (9.0 to $9.4 \mathrm{mg}$ ) were weighed into $50 \mu \mathrm{l}$ aluminium pans, and covers were sealed into place. The instrument was calibrated with reference to the enthalpy and the melting points of indium (melting temperature $156.6^{\circ} \mathrm{C} ; \Delta \mathrm{H} 28.45 \mathrm{~J} / \mathrm{g}$ ), and decane (melting temperature $-29.7^{\circ} \mathrm{C}$;

$\Delta \mathrm{H} 202.1 \mathrm{~J} / \mathrm{g}$ ) standards. An empty pan was used for reference. Dry nitrogen was used as purge gas in the DSC cell at $20 \mathrm{~cm}^{3} / \mathrm{min}$. Thermograms were analyzed using Pyris Software to obtain the enthalpy $\left(\mathrm{J} / \mathrm{g}\right.$, integration of the DSC signals) and $\mathrm{T}_{\text {onset }}$ and $\mathrm{T}_{\text {end }}$ of the transformations $\left({ }^{\circ} \mathrm{C}\right.$, intersections of the baseline and the initial and final tangents at the transformation). A correction (described elsewhere $^{30}$ ) was applied for analysis with cooling or heating rates other than $2^{\circ} \mathrm{C} \cdot \mathrm{min}^{-1}$, since the calorimeter was calibrated at this rate.

Laboratory-scale powder XRD allowed identifying the crystal forms present by comparison to previous work, in which characteristic XRD patterns were reported. The measurements were performed using a PANalytical X'Pert Pro MPD powder diffractometer equipped with a Hybrid Monochromator and an X'Celerator Detector. The equipment also included an Oxford Cryostream Plus 220V (temperature 80 to $500 \mathrm{~K}$ ). This diffractometer was operated with DebyeScherrer transmission. The sample was introduced in a 1mm-diameter Lindemann glass capillary. The latter was rotated about its axis during the experiment to minimize preferential orientations of the crystallites. The step size was $0.013^{\circ}$ from $1.004^{\circ}$ to $28^{\circ} 2 \theta$.

Three independent DSC aluminium pans and three XRD capillaries were prepared for each mixture to thermodynamically stabilize the binary-mixture samples. All DSC pans and XRD capillaries were rapidly cooled to $-20^{\circ} \mathrm{C}$ and then tempered at different temperatures in order to 
achieve equilibration. Thus, samples were subjected to incubation in order to attempt to detect more stable forms. Due to the uncertainty of the necessary time to stabilize the samples, we incubated three independent DSC pans and XRD capillaries in order to make some control analysis within the stabilization period. By incubating three DSC pans and XRD capillaries, we ensured consistent results with the objectives of our study. We selected temperatures close to the melting point of least stable polymorphic forms, but at which the entire sample was still in solid state. The incubation temperatures used were (i) $17^{\circ} \mathrm{C}$ for PPO, and $10^{\circ} \mathrm{C}$ for OOP and OPO; (ii) $6^{\circ} \mathrm{C}$ for all OOP-OPO mixtures; (iii) $10^{\circ} \mathrm{C}$ for PPO-OOP mixtures from 10PPO:90OOP to 50PPO:50OOP, and $17^{\circ} \mathrm{C}$ for PPO-OOP mixtures from 60PPO:40OOP to 90PPO:10OOP; and (iv) $10^{\circ} \mathrm{C}$ for all PPO-OPO mixtures. Samples were kept under these conditions for 17 months. This incubation period was determined in an empirical way.

The transformation and melting behavior of the incubated mixtures were measured using DSC by heating the samples from $0^{\circ} \mathrm{C}$ to $50^{\circ} \mathrm{C}$ at $2^{\circ} \mathrm{C} \cdot \mathrm{min}^{-1}$. Shape factors $\Delta \mathrm{T}_{\text {end }}{ }^{31}$ were calculated measuring $\left|\mathrm{T}_{\text {onset }}-\mathrm{T}_{\text {end }}\right|$ of the melting signal of the TAG components and used to obtain the characteristic temperatures of the complex phenomena of phase behavior.

Kinetic experiments were performed on the 50OOP:50OPO and 50PPO:50OOP binary mixtures in order to observe metastable forms of MC. We selected the incubation temperatures of some of the binary mixtures using the data on the melting behavior of the metastable forms. Isothermal processes (at $10^{\circ} \mathrm{C}$ and $0^{\circ} \mathrm{C}$ ) and dynamic temperature variations were applied to the samples. For the dynamic experiments, samples were cooled from $35^{\circ} \mathrm{C}$ to $-30^{\circ} \mathrm{C}$ at different cooling rates $\left(15,2\right.$, and $\left.0.5^{\circ} \mathrm{C} \cdot \mathrm{min}^{-1}\right)$ and heated from $-30^{\circ} \mathrm{C}$ to $35^{\circ} \mathrm{C}$ at $2^{\circ} \mathrm{C} \cdot \mathrm{min}^{-1}$.

Synchrotron Radiation X-Ray Diffraction (SR-XRD) with Small-Angle X-Ray Diffraction (SAXD) and Wide-Angle X-Ray Diffraction (WAXD) was used for some experiment conditions. These SR-XRD experiments were performed at BL-6A of the synchrotron radiation facility Photon Factory (PF) at the High-Energy Accelerator Research Organization (KEK) in Tsukuba (Japan). A double-focusing camera was operated at a wavelength of $0.15 \mathrm{~nm}$. The X-ray 
scattering data were simultaneously collected using a CCD camera for small-angle data and a Pilatus-100K detector for wide-angle data. The temperature program was controlled using a Mettler DSC-FP84 with FP99 software. A 2mm-thick sample was placed in an aluminium sample cell with Kapton film windows. SR-XRD spectra were acquired at 30s intervals.

\author{
RESULTS \\ Binary phase behavior of 1,2-dioleoyl-3-palmitoyl glycerol and 1,3-dioleoyl-2-palmitoyl \\ glycerol (OOP-OPO)
}

\title{
Occurrence of OOP:OPO molecular compound (non-incubated samples)
}

In order to observe MC formation at the ratio of 5000P:50OPO, different thermal treatments of isothermal processes and cooling experiments at different rates $\left(15,2\right.$, and $\left.0.5^{\circ} \mathrm{C} \cdot \mathrm{min}^{-1}\right)$ were conducted. We observed the polymorphic occurrence of metastable forms of the 50OOP:50OPO binary mixture.

Figure 2 depicts the polymorphic behavior of 50OOP:50OPO when it was cooled from $35^{\circ} \mathrm{C}$ to $-35^{\circ} \mathrm{C}$ at a rate of $0.5^{\circ} \mathrm{C} \cdot \mathrm{min}^{-1}$ and heated at $2^{\circ} \mathrm{C} \cdot \mathrm{min}^{-1}$. 


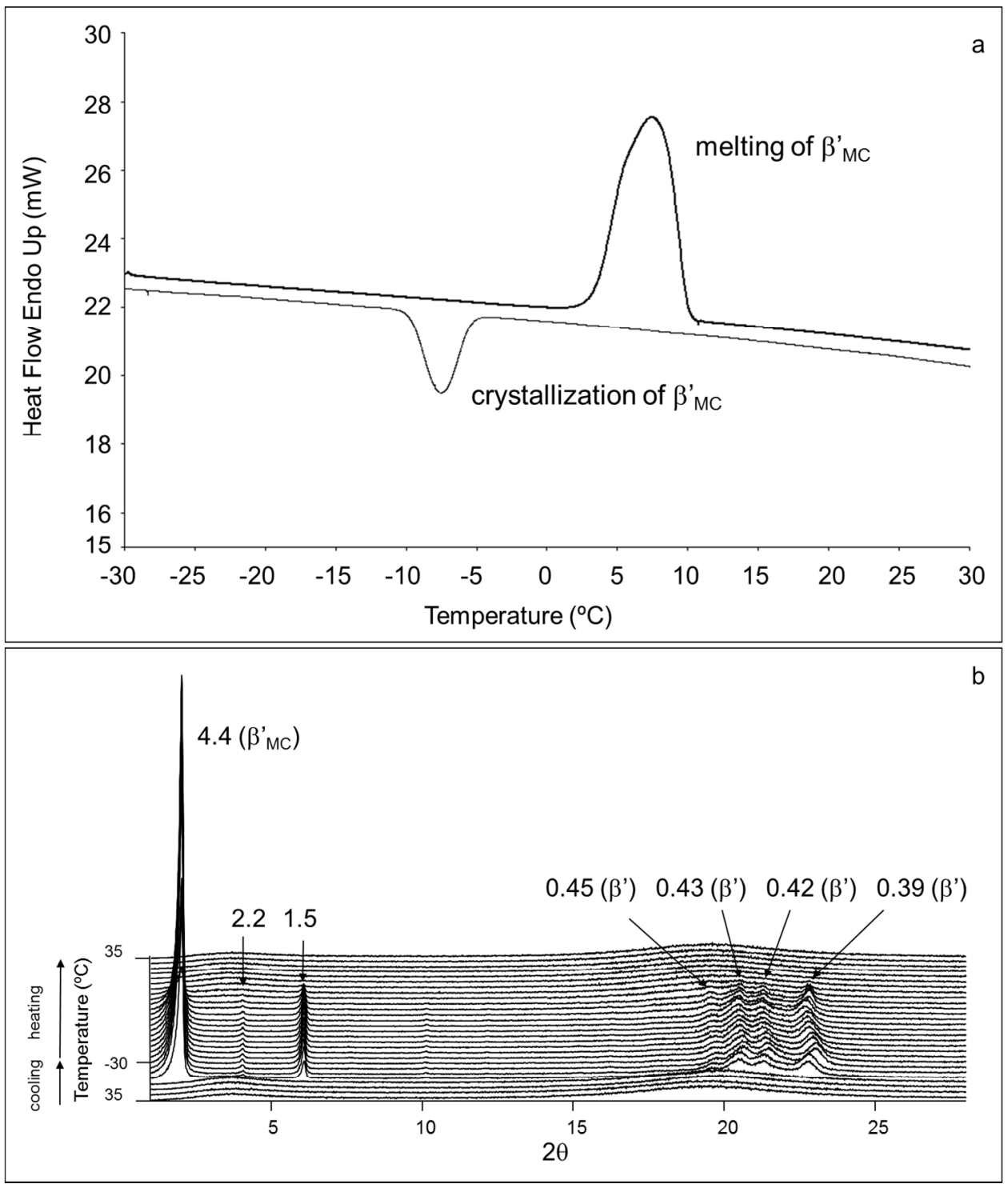

Figure 2. Polymorphic behavior of metastable forms of 500OP:50OPO when cooled at $0.5^{\circ} \mathrm{C} \cdot \mathrm{min}^{-1}$ and heated at $2^{\circ} \mathrm{C} \cdot \mathrm{min}^{-1}$. a) DSC thermogram. b) XRD paterns.

When 50OOP:50OPO was cooled at $0.5^{\circ} \mathrm{C} \cdot \mathrm{min}^{-1}$, a single exothermic DSC peak with $\mathrm{T}_{\text {onset }}$ at $5.4^{\circ} \mathrm{C}$ appeared in the cooling curve (Fig. 2a). According to the XRD data (Fig. 2b), this peak is due to $\beta^{\prime}$ оop:Opo crystallization, as a double-chain-length structure SAXD peak at $4.4 \mathrm{~nm}$ and WAXD peaks at $0.45 \mathrm{~nm}, 0.43 \mathrm{~nm}, 0.42 \mathrm{~nm}$, and $0.39 \mathrm{~nm}$ were identified. During heating, only one 
broad endothermic DSC peak appeared, with $\mathrm{T}_{\text {onset }}$ of $3.4^{\circ} \mathrm{C}$ and $\mathrm{T}_{\text {end }}$ of $10.0^{\circ} \mathrm{C}$, which corresponded to $\beta^{\prime}$ OOP:OPO melting.

The same results were obtained using a cooling and heating rate of $2^{\circ} \mathrm{C} \cdot \mathrm{min}^{-1}$. However, the least stable sub- $\alpha$ form of the OOP:OPO molecular compound was crystallized when a high cooling rate $\left(15^{\circ} \mathrm{C} \cdot \mathrm{min}^{-1}\right)$ was applied to the sample (data not shown).

Formation of a OOP:OPO molecular compound at the ratio of 50:50 was confirmed, and two polymorphic forms (sub- $\alpha_{\mathrm{OOP}: \mathrm{OPO}}$ and $\beta^{\prime}{ }_{\mathrm{OOP}: \mathrm{OPO}}$ ) were detected by applying different thermal treatments to the 50OOP:50OPO binary mixture.

\section{Phase behavior of incubated OOP-OPO binary mixtures}

DSC and laboratory-scale XRD experiments were performed after 17 months of incubation of the samples to analyze the phase behavior in the most stable state of the OOP-OPO mixtures. Figure 3 illustrates the DSC and XRD data obtained at different concentration ratios. DSC thermograms were registered while heating from $0^{\circ} \mathrm{C}$ to $50^{\circ} \mathrm{C}$ at a rate of $2^{\circ} \mathrm{C} \cdot \mathrm{min}^{-1}$, whereas XRD experiments were performed at $5^{\circ} \mathrm{C}$. 

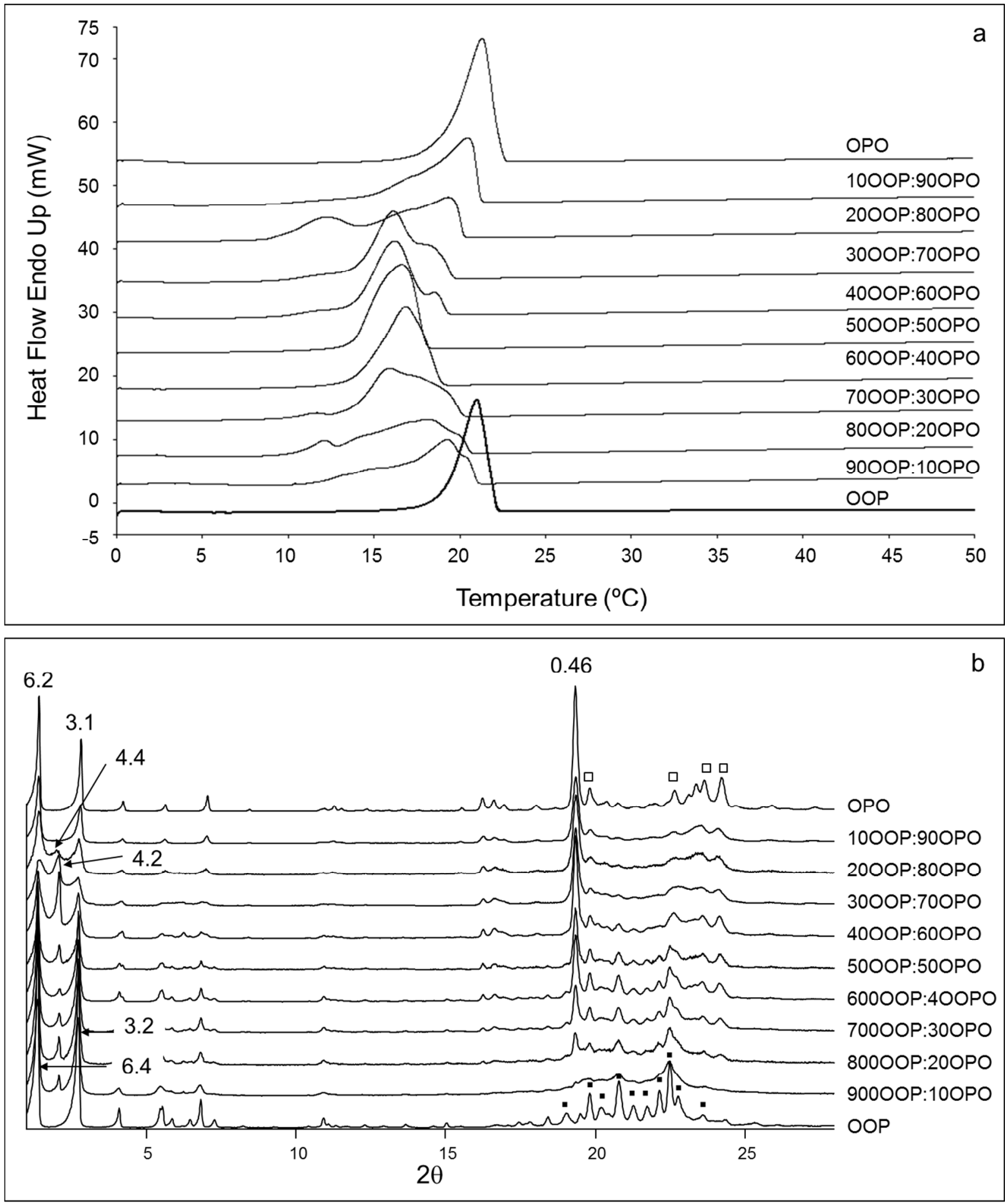

Figure 3. a) DSC heating thermopeaks of OOP-OPO mixtures. b) XRD paterns of OOP-OPO mixtures at $5^{\circ} \mathrm{C}$. Data were obtained after an incubation period of 17 months. 
The DSC heating curves of the two TAG components exhibited only the melting peak of their most stable form. The corresponding XRD patterns exhibited clear patterns of $\beta_{\mathrm{OPO}}$ and $\beta$ ' ${ }_{\mathrm{OOP}}$. $\beta_{\text {OPO }}$, having a triple-chain-length structure $(6.2 \mathrm{~nm}$ and $3.1 \mathrm{~nm})$ was identified by the typical $\beta$ form intense peak at $0.46 \mathrm{~nm}$ and other wide-angle diffraction peaks at $0.44 \mathrm{~nm}, 0.39 \mathrm{~nm}, 0.38 \mathrm{~nm}$, and $0.37 \mathrm{~nm}$ (denoted by $\square$ in Fig. 3b). The XRD pattern of incubated OOP exhibited triplechain-length peaks, with long spacings of $6.4 \mathrm{~nm}$ and $3.2 \mathrm{~nm}$, and wide-angle diffraction peaks at $0.47 \mathrm{~nm}, 0.45 \mathrm{~nm}, 0.44 \mathrm{~nm}, 0.43 \mathrm{~nm}, 0.42 \mathrm{~nm}, 0.41 \mathrm{~nm}, 0.40 \mathrm{~nm}$, two peaks at $0.39 \mathrm{~nm}$, and $0.38 \mathrm{~nm}$ (denoted by $\cdot$ in Fig. 3b).

At least two endothermic peaks were observed in the DSC heating curves of mixtures with 10:90 to 40:60 concentration ratios. In some cases, another endothermic peak appeared at $9^{\circ} \mathrm{C}$ ( $\mathrm{T}_{\text {onset }}$ ), becoming stronger in the $20: 80$ composition. A single broad endothermic peak with $\mathrm{T}_{\text {onset }}$ at $14^{\circ} \mathrm{C}$ was present in 50:50 and 60:40 compositions, and three or more melting peaks were observed in the DSC thermograms with $70: 30$ to $90: 10$ concentration ratios. Similarly to the 20:80 composition, we observed an endothermic signal at $\mathrm{T}_{\text {onset }}$ of $9^{\circ} \mathrm{C}$ at 70:30 and 80:20 concentration ratios. XRD patterns indicated the presence of a triple-chain-length structure at all concentration ratios. Moreover, double-chain-length peaks were observed in the short-angle regions from 20:80 to 90:10 concentration ratios. In almost all cases, the corresponding long spacing was $4.2 \mathrm{~nm}$, except for the 20:80 binary mixture, where a spacing of $4.4 \mathrm{~nm}$ was detected. The intensity of the $\beta$ diffraction peak at $0.46 \mathrm{~nm}$ decreased as the OOP composition increased.

Laboratory-scale XRD experiments were performed as a function of temperature for some of the incubated compositions in order to understand the complicated phase behavior of incubated OOP-OPO binary mixtures (data not shown). 
The results demonstrated that the XRD peak at 4.4nm in the 20OOP:80OPO composition

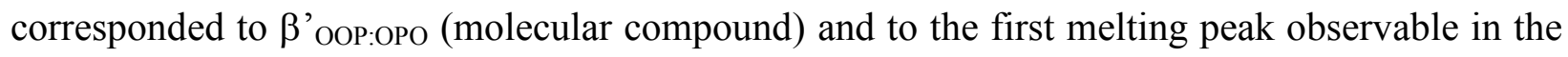
DSC thermogram of the 20:80 binary mixture (Fig. 3). This peak did not exist, or at least was weaker, at the other concentration ratios.

With the 50OOP:50OPO binary mixture, the XRD peaks indicated the presence of a doublechain-length structure, corresponding to the OOP:OPO molecular compound, as well as a triplechain-length structure at $6.4 \mathrm{~nm}$ and $3.1 \mathrm{~nm}$ (Fig. 3b). The occurrence of OOP:OPO molecular compound was confirmed by kinetic study (Fig. 2). However, from the results obtained after 17 months of incubation, we may conclude that incubation caused partial decomposition of the molecular compound into two TAG components. Only one endothermic peak appeared in the corresponding DSC heating curve (Fig. 3b), although we should consider the width of the peak. The long spacing corresponding to the double-chain-length structure was $4.2 \mathrm{~nm}$, which differed from that of the 20:80 composition $(4.4 \mathrm{~nm})$. These results confirmed that the long spacing of $4.2 \mathrm{~nm}$ corresponded to $\beta_{\mathrm{OOP}: \mathrm{OPO}}$. Hence, $\beta$ ' ${ }_{\text {OOP:OPO }}$ observed at the $20: 80$ concentration ratio became a metastable form of the molecular compound, with $\beta_{\mathrm{OOP}: \mathrm{OPO}}$ being the most stable one.

Based on these results, we may conclude that a OOP:OPO molecular compound definitely formed, and several polymorphic forms were detected. Studying this molecular compound by changing the cooling rates permitted us to identify two different polymorphic forms: sub-

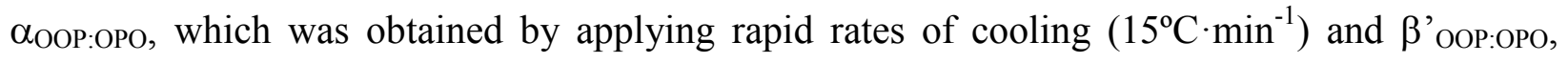
which crystallized at intermediate $\left(2^{\circ} \mathrm{C} \cdot \mathrm{min}^{-1}\right)$ and low $\left(0.5^{\circ} \mathrm{C} \cdot \mathrm{min}^{-1}\right)$ cooling rates. Moreover, thermodynamic stabilization for 17 months at $10^{\circ} \mathrm{C}$ caused the molecular compound to evolve to the most stable $\beta_{\text {OOP:OPO. }}$ 
However, we should consider the presence of some metastable $\beta$ ' ${ }_{\text {OOP:OPO, which did not }}$ completely transform to $\beta_{\mathrm{OOP}: \mathrm{OPO}}$ at some of the concentration ratios after incubation. The most surprising fact was the presence of triple-chain-length structures of pure TAG components ( $\beta$ ' and $\left.\beta_{\mathrm{OPO}}\right)$ at the $50: 50$ concentration ratio after 17 months of incubation, demonstrating that the molecular compound should finally be metastable and tended to decompose into pure TAG components during stabilization.

Additional XRD experiments were performed after 6 and 11 months of incubation in order to monitor binary mixture stabilization. At different compositions we observed a progressive increase of the intensity of triple-chain-length diffraction peaks of the TAG components ( $\beta$ ' ${ }_{\text {OOP }}$ and $\left.\beta_{\mathrm{OPO}}\right)$ at the expense of the double-chain-length diffraction peak, corresponding to the OOP:OPO molecular compound ( $\left.\beta_{\mathrm{OOP}: \mathrm{OPO}}\right)$. This fact confirms the metastable nature of the OOP:OPO molecular compound. Thus, the first polymorphic form of the OOP:OPO molecular

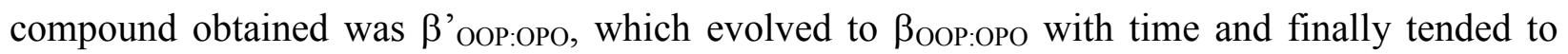
separate into two TAG components. However, the evolution from MC to two TAG components was slow, as confirmed by the XRD data obtained for the 50OOP:50OPO binary mixture after 11 and 17 months of incubation (Fig. S1, Supplementary Materials), where no significant differences could be detected. Moreover, the incubation period of 17 months was not long enough for $\mathrm{MC}$ to completely disappear. We determined the tendency of $\mathrm{MC}$ to separate into component TAGs, although it is not possible to make an estimation of the time needed to observe the complete vanishing of $\mathrm{MC}$. 


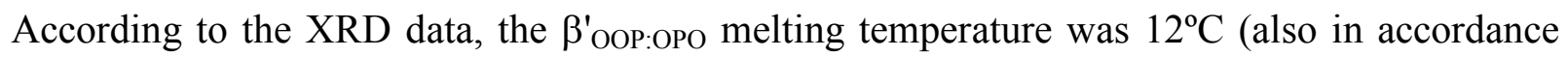
with $\mathrm{T}_{\text {onset }}$ of the corresponding DSC thermogram), and the $\beta_{\mathrm{OOP}: \mathrm{OPO}}$ melting temperature was $17^{\circ} \mathrm{C}$.

OOP:OPO molecular compound was observed in the metastable state and the system tended to an eutectic equilibrium between stable forms of the component TAGs. Figure 4 illustrates the phase behavior of OOP-OPO mixtures.

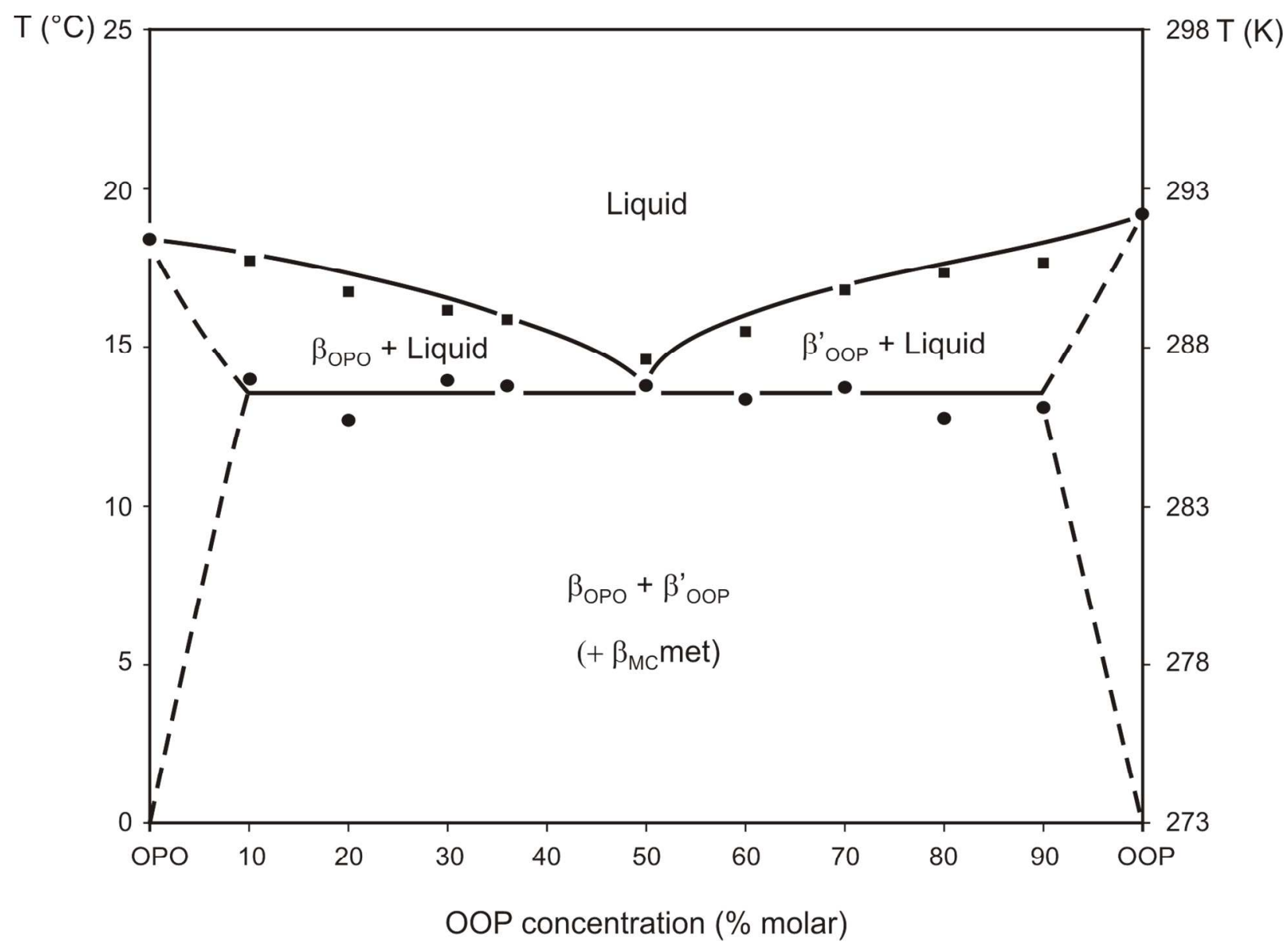


Figure 4. Phase behavior of OOP-OPO mixtures, based on DSC onset and end temperatures $\left(\mathrm{T}_{\text {end }}-\Delta \mathrm{T}_{\text {end }}\right)$. Dashed lines represent solubility domain limits, which were defined by extrapolation, not by precise experimental determination.

According to the DSC heating curves (Fig. 3a), the eutectic point should be located at the 50OOP:50OPO composition. As already stated, the melting temperature of the most stable $\beta$ form of the OOP:OPO molecular compound was around $17^{\circ} \mathrm{C}$, which is above the eutectic point.

Binary phase behavior of 1,2-dipalmitoyl-3-oleoyl glycerol and 1,2-dioleoyl-3-palmitoyl glycerol (PPO-OOP)

\title{
Occurrence of PPO:OOP molecular compound (non-incubated samples)
}

\begin{abstract}
Several thermal treatments (isothermal and dynamic processes) were applied to the 50PPO:50OOP mixture in order to observe the MC crystals of PPO-OOP in the metastable state.
\end{abstract} Figures 5 and 6 illustrate the polymorphic behavior observed when the 50PPO:50OOP mixture was cooled and heated at $2^{\circ} \mathrm{C} \cdot \mathrm{min}^{-1}$. 


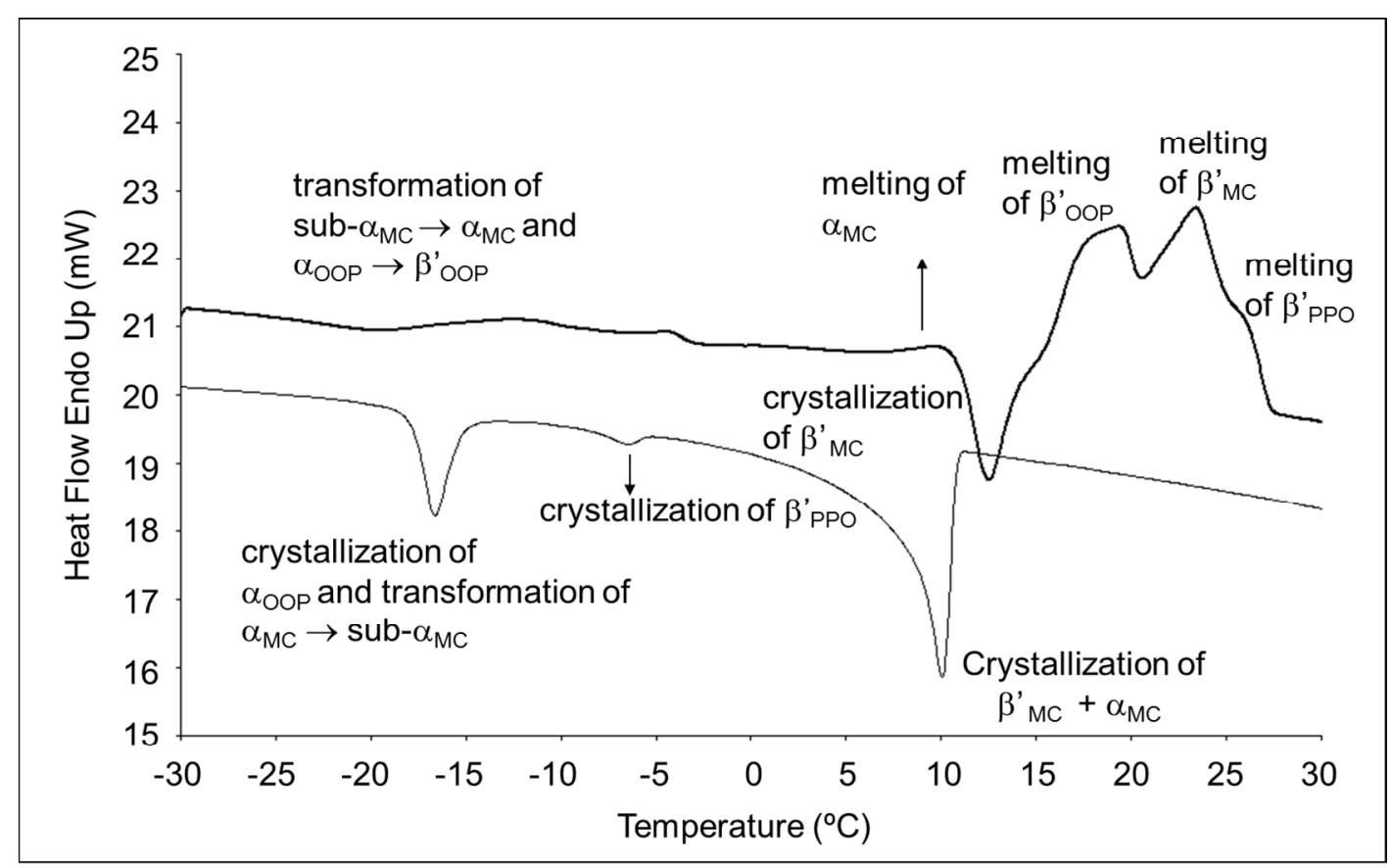

Figure 5. DSC cooling and heating thermopeaks of metastable forms of 50PPO:50OOP mixture obtained during cooling and heating processes at a rate of $2^{\circ} \mathrm{C} \cdot \mathrm{min}^{-1}$.

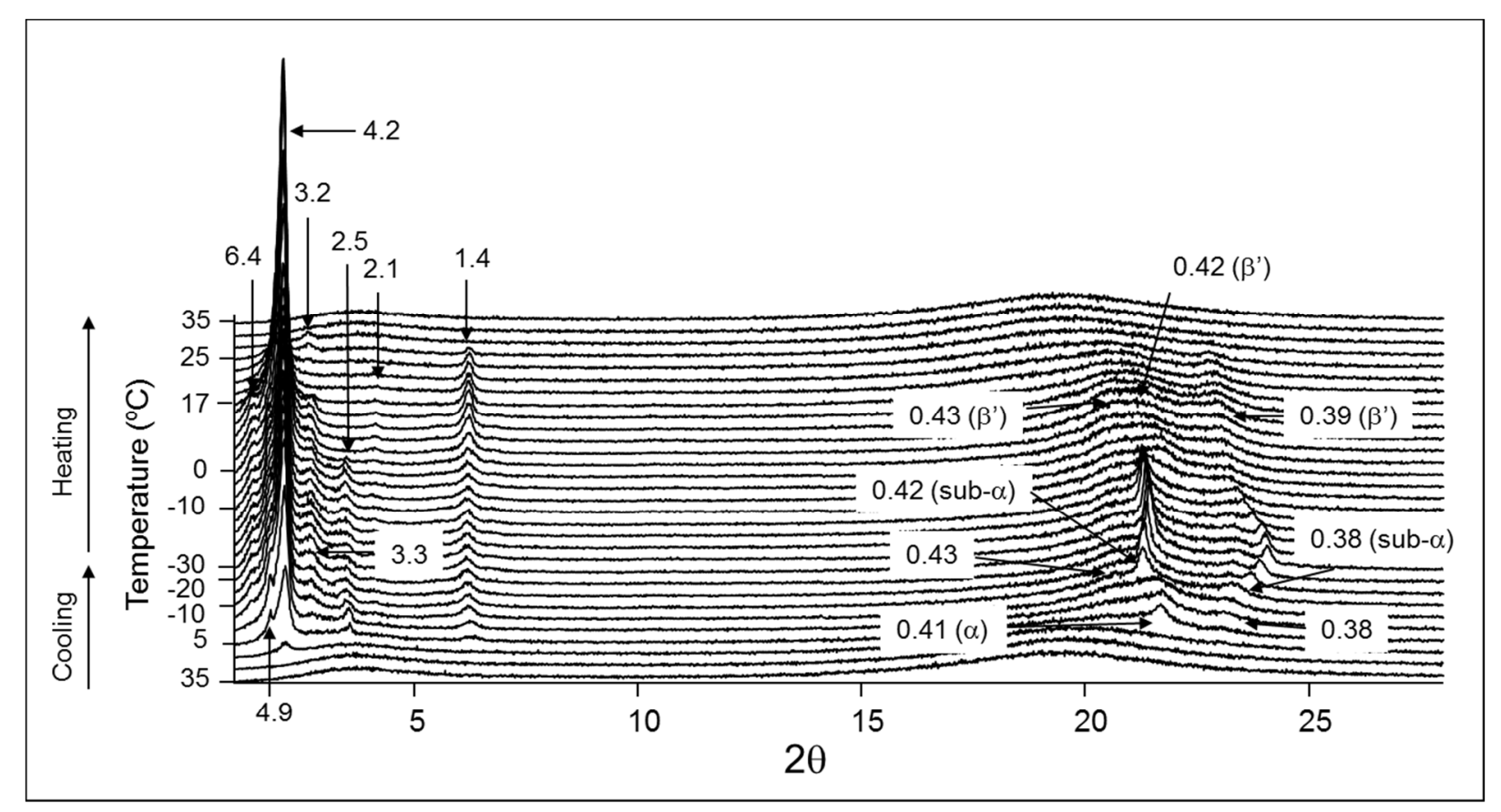


Figure 6. XRD paterns of metastable forms of 50PPO:50OOP mixture obtained during cooling and heating processes at a rate of $2^{\circ} \mathrm{C} \cdot \mathrm{min}^{-1}$.

More complex polymorphic behavior was observed in 50PPO:50OOP mixture compared to that of 50OOP:50OPO. The XRD data indicated the first occurring XRD peak with a long spacing of $4.2 \mathrm{~nm}$ (Fig. 6). Soon after, another peak at $4.9 \mathrm{~nm}$ appeared in the short-angle region. The wide-angle region exhibited a typical $\alpha$ form XRD peak at $0.41 \mathrm{~nm}$ accompanied by a broad peak at $0.38 \mathrm{~nm}$. Therefore, the first crystallizing form should be $\beta^{\prime}{ }_{\mathrm{PPO}: \mathrm{OOP}}$, and $\alpha_{\mathrm{PPO}: \mathrm{OOP}}$ immediately formed from the melt. The broad profile of the wide-angle XRD region indicated the coexistence of both polymorphic forms. This complex crystallization process appeared in the DSC thermogram (Fig. 5) as a broad exothermic peak with $\mathrm{T}_{\text {onset }}$ of $10.8^{\circ} \mathrm{C}$. On further cooling, another DSC peak was observed at $\mathrm{T}_{\text {onset }}$ of $-4.9^{\circ} \mathrm{C}$. This should correspond to $\beta^{\prime}{ }_{\mathrm{PPO}}$ crystallization, as triple-chain structure XRD peaks at $6.4 \mathrm{~nm}$ and $3.3 \mathrm{~nm}$ and a new $\beta$ ' peak at $0.43 \mathrm{~nm}$ were observed in the XRD patterns. It should be noted that the wide-angle region peaks became broader because of overlapping signals. Another exothermic phenomenon appeared at $\mathrm{T}_{\text {onset }}$ of $-9.8^{\circ} \mathrm{C}$ in the DSC cooling curve. The XRD data indicated the presence of sharp sub- $\alpha$ peaks at $0.42 \mathrm{~nm}$ and $0.38 \mathrm{~nm}$. Thus, $\alpha_{\mathrm{PPO}: \mathrm{OOP}}$ may have transformed to sub- $\alpha_{\mathrm{PPO}: \mathrm{OOP}}$, and $\alpha_{\mathrm{OOP}}$ may have crystallized. As previous experiments demonstrated, ${ }^{29} \alpha_{\mathrm{OOP}}$ crystallized from the melt at $-11.3^{\circ} \mathrm{C}\left(\mathrm{T}_{\text {onset }}\right)$ when the sample was cooled at a rate of $2^{\circ} \mathrm{C} \cdot \mathrm{min}^{-1}$.

When the 50PPO:50OOP mixture was heated from -30 to $35^{\circ} \mathrm{C}$ at $2{ }^{\circ} \mathrm{C} \cdot \mathrm{min}^{-1}$, the DSC curve exhibited a series of complex phenomena at temperatures from -20 to $-5^{\circ} \mathrm{C}$ (DSC peaks in Fig. 5). The corresponding XRD patterns indicated the disappearance of wide-angle sub- $\alpha$ peaks (the peak at $0.38 \mathrm{~nm}$ vanished), so sub- $\alpha_{\mathrm{PPO}: \mathrm{OOP}}$ may have transformed to $\alpha_{\mathrm{PPO}: \mathrm{OOP}}$, and broad $\beta$ ' peaks 
$(0.43 \mathrm{~nm}, 0.42 \mathrm{~nm}$, and $0.39 \mathrm{~nm})$ began to appear, which gave an indication of a $\alpha_{\mathrm{OOP}} \rightarrow \beta^{\prime}{ }_{\mathrm{OOP}}$ transformation. Further on, a melt-mediated transformation was observed in the DSC heating thermogram, consisting of $\alpha_{\mathrm{PPO}: \mathrm{OOP}}$ melting (with $\mathrm{T}_{\text {onset }}$ at $7.2^{\circ} \mathrm{C}$ ) and subsequent $\beta^{\prime}{ }_{\mathrm{PPO}: \mathrm{OOP}}$ crystallization (with $\mathrm{T}_{\text {onset }}$ at $10.6^{\circ} \mathrm{C}$ ). Simultaneously, the XRD patterns indicated the disappearance of short-angle region peaks at $4.9 \mathrm{~nm}$ and $2.5 \mathrm{~nm}$, corresponding to $\alpha_{\mathrm{PPO}: \mathrm{OOP}}$, and $\beta^{\prime}$ peaks at $0.43 \mathrm{~nm}, 0.42 \mathrm{~nm}$, and $0.38 \mathrm{~nm}$ that became more defined. Finally, the DSC heating curve exhibited complicated melting behavior consisting of the melting of $\beta^{\text {' }}$ oop with a peak top temperature of $19.4^{\circ} \mathrm{C}, \beta{ }^{\prime}{ }_{\mathrm{PPO}: \mathrm{OOP}}$ with a peak top temperature of $23.4^{\circ} \mathrm{C}$, and $\beta{ }^{\prime}{ }_{\mathrm{PPO}}$ with a peak top temperature of $26.1^{\circ} \mathrm{C}$. Simultaneously, the XRD patterns indicated the disappearance of triple-chain-length diffraction peaks at $6.4 \mathrm{~nm}$ and $3.3 \mathrm{~nm}$. Later, a new triple-chain-length at $3.2 \mathrm{~nm}$ could be observed in the short-angle region, which vanished simultaneously with the double-chain-length peak at $4.2 \mathrm{~nm}$. It is unclear why the new peak at $3.2 \mathrm{~nm}$ appeared on heating at such high temperatures, just before melting. Some additional crystallization may have occurred during heating.

In order to clarify the polymorphic behavior of 50PPO:50OOP, a shorter thermal treatment was performed when the binary mixture was cooled and heated at $2^{\circ} \mathrm{C} \cdot \mathrm{min}^{-1}$ (data not shown). Thus, the sample was cooled from 35 to $0^{\circ} \mathrm{C}$ (instead of $-30^{\circ} \mathrm{C}$ ) and then heated to $35^{\circ} \mathrm{C}$. The first exothermic DSC peak with $\mathrm{T}_{\text {onset }}$ at $10.8^{\circ} \mathrm{C}$ was observed. When heating, melt-mediated transformation, consisting of melting at $7.2^{\circ} \mathrm{C}$ and subsequent crystallization at $10.6^{\circ} \mathrm{C}$, also occurred. However, on further heating, only one melting peak appeared. These results demonstrated that melt-mediated transformation was related to $\alpha_{\mathrm{PPO}: \mathrm{OOP}}$ and $\beta_{\mathrm{PPO}}^{\text {(OOP }}$, which firstly occurred during the cooling process. This confirmed the melt-mediated transformation from $\alpha_{\text {PPO:OOP }}$ to $\beta^{\prime}$ PPO:OOP. 
To summarize, the PPO-OOP binary mixtures were MC-forming, and several polymorphic forms of the molecular compound (sub- $\alpha_{\mathrm{PPO}: \mathrm{OOP}}, \alpha_{\mathrm{PPO}: \mathrm{OOP}}$, and $\beta_{\text {PPO:OOP}}$ ) were determined by studying the metastable phases of the 50PPO:50OOP composition when it was subjected to different thermal treatments. However, unlike that with OOP:OPO, not all the sample was composed of the PPO:OOP molecular compound; with thermal treatments applied, the coexistence of MC and pure TAG components was observed.

\section{Phase behavior of incubated PPO-OOP binary mixtures}

Further DSC and XRD experiments were carried out after 17 months of stabilizing the binary mixtures. Figure 7 depicts the heating thermograms obtained at a rate of $2^{\circ} \mathrm{C} \cdot \mathrm{min}^{-1}$ (Fig. 7a) and $\mathrm{XRD}$ analysis conducted at $5^{\circ} \mathrm{C}$ (Fig. 7b) for PPO-OOP mixtures at various concentration ratios.

Single endothermic peaks were observed for pure OOP and PPO, corresponding to the melting of $\beta$ ' form of the two TAGs. The XRD data indicated triple-chain-length structure peaks, with long spacings of $6.5 \mathrm{~nm}$ and $3.2 \mathrm{~nm}$. Typical $\beta$ ' form peaks were observed in the wide-angle diffraction region. The short spacings of $\beta$ ' ${ }_{\text {OOP }}$ (denoted by $\square$ in Fig. $4 \mathrm{~b}$ ) were $0.47 \mathrm{~nm}, 0.45 \mathrm{~nm}$, $0.44 \mathrm{~nm}, 0.43 \mathrm{~nm}, 0.42 \mathrm{~nm}, 0.41 \mathrm{~nm}, 0.40 \mathrm{~nm}, 0.39 \mathrm{~nm}, 0.39 \mathrm{~nm}$, and $0.38 \mathrm{~nm}$. However, typical

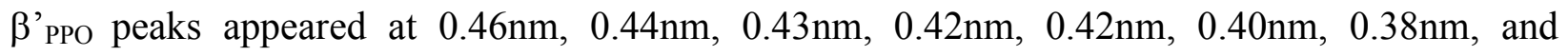
$0.37 \mathrm{~nm}$ (denoted by $\cdot$ ) in the wide-angle region. Two endothermic DSC peaks appeared in the PPO-OOP mixtures at 10:90 and 20:80 concentration ratios, although the first endothermic peak of the 20:80 composition was considerably broad, and the second one was weak. The DSC heating curves of the 30:70,40:60, and 50:50 concentration ratios became highly complex, indicating more than three endothermic DSC peaks in all cases. Concurrently, XRD patterns 
corresponding to the OOP-rich region (from 10PPO:90OOP to 40PPO:60OOP) and the 50PPO:50OOP mixture exhibited broad diffraction peaks in the wide-angle region, probably due to the overlapping of several polymorphic forms. Furthermore, from the 20PPO:80OOP to the 50PPO:50OOP composition, a double-chain-length diffraction peak appeared in the short-angle region, with a spacing of $4.2 \mathrm{~nm}$. 

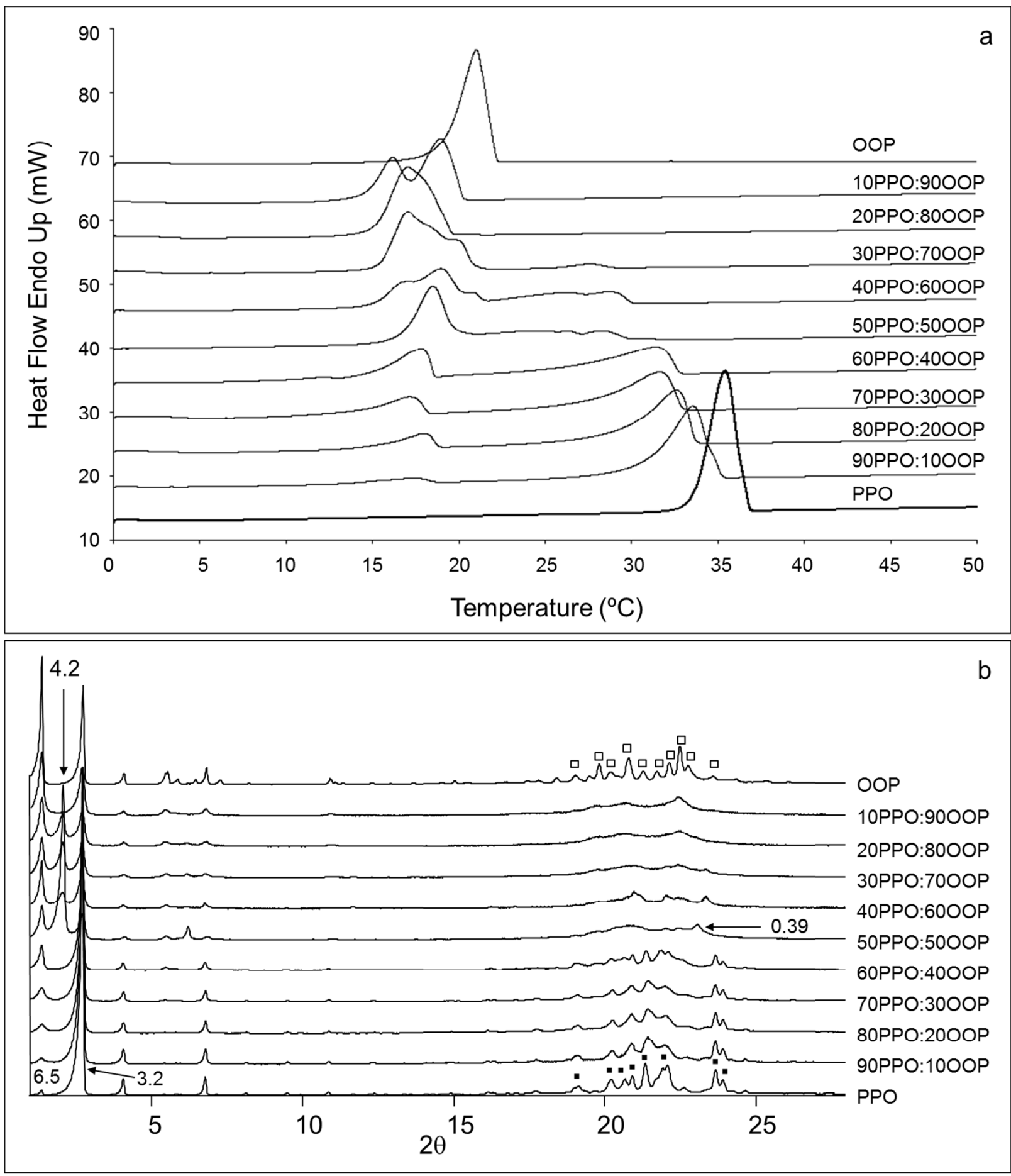

Figure 7. a) DSC heating thermopeaks of PPO-OOP mixtures. b) XRD paterns of PPO-OOP mixtures at $5^{\circ} \mathrm{C}$. Data were obtained after an incubation period of 17 months. 
Nevertheless, two well-defined melting DSC peaks were observed in the PPO-rich region, where $T_{\text {onset }}$ of the first melting peak was maintained and $T_{\text {end }}$ of the second peak increased with increasing PPO concentration. The corresponding XRD patterns exhibited clear $\beta$ ' diffraction peaks, and no double-chain-length peak at $4.2 \mathrm{~nm}$ was present in the entire PPO-rich region.

Hence, in a manner similar to that used for the OOP-OPO system, laboratory-scale XRD was carried out as a function of temperature for some binary mixtures in order to investigate the phenomena observed in the DSC thermograms. The behavior observed in the OOP-rich region differed from that of the PPO-rich region. Furthermore, equilibrium between $\mathrm{MC}_{\mathrm{PPO}: \mathrm{OOP}}$ and pure TAG components was not present at all concentration ratios of the PPO-OOP mixtures. As shown in Figure 7, the double-chain-length diffraction peak at $4.2 \mathrm{~nm}$ corresponding to the $\mathrm{MC}_{\mathrm{PPO}: \mathrm{OOP}}$ ( $\beta^{\prime}$ form) was only detected in the binary mixtures from 20PPO:80OOP to 50PPO:50OOP and was more intense with the latter ratio. For the DSC heating profiles, more complicated melting behavior was observed in the OOP-rich region (due to the presence of metastable phases), whereas the thermograms of the PPO-rich region consisted of only two endothermic peaks. Thus, the mixing behavior of the OOP-rich region seemed to be MCforming, whereas the PPO-rich region followed a eutectic pattern.

At this point, it was necessary to determine the reason for such different behaviors in the two compositional regions. The results shown above were obtained from samples which were kept for 17 months at different incubation temperatures, mixtures from 10PPO:90OOP to 50PPO:50OOP were incubated at $10^{\circ} \mathrm{C}$, and concentration ratios from 60PPO:40OOP to 90PPO:10OOP were incubated at $17^{\circ} \mathrm{C}$. Therefore, we decided to incubate new samples of pure PPO and binary mixtures from 60PPO:400OP to 90PPO:100OP at $10^{\circ} \mathrm{C}$ for three months, to check whether incubation at $10^{\circ} \mathrm{C}$ and $17^{\circ} \mathrm{C}$ of the PPO-rich region may result in a different 
behavior. The same DSC heating curves were obtained when incubation temperature was $10^{\circ} \mathrm{C}$ and $17^{\circ} \mathrm{C}$, indicating that the incubation temperature was not responsible for the different behaviors observed in the two compositional regions.

Additional XRD experiments were carried out after 6 and 11 months of incubation, so we could compare the data obtained with that after 17 months of incubation. As a consequence, we realized that for some compositions the triple-chain-length diffraction peaks at $6.5 \mathrm{~nm}$ (corresponding to $\beta^{\prime}{ }_{\mathrm{OOP}}$ ) and at $3.2 \mathrm{~nm}$ (corresponding to $\beta^{\prime}{ }_{\mathrm{PPO}}$ ) increased with time at the expense of the double-chain-length diffraction peak at $4.2 \mathrm{~nm}$ (corresponding to $\beta$ ' ${ }_{\mathrm{PPO}} \mathrm{OOP}$ ). We observed a difference in the relative intensity of the triple and double-chain-length diffraction peaks soon after the 50PPO:50OOP composition crystallized (Fig. 6) and after 17 months of incubation (Fig. 7b). Before the sample was incubated, triple-chain-length diffraction peaks became weaker than those observed after stabilization. Thus, we concluded that the MC formed ( $\beta$ ' ${ }_{\text {PPO:OOP) }}$ was metastable and tended to disappear, being separated into two $\beta$, TAG components during thermodynamic stabilization of the samples, similar to OOP-OPO. In other words, MC, with melting point of $19^{\circ} \mathrm{C}$ ( $\left.\beta^{\prime}{ }_{\mathrm{PPO}} \mathrm{OOP}\right)$, formed initially; however, due to its metastable nature, it separated into two $\beta$, TAG components, indicating a eutectic behavior. In Figure $7 \mathrm{a}$, from the 10PPO:90OOP to the 90PPO:10OOP composition, one may notice a constant onset temperature, which indicates the eutectic invariant. The liquidus temperature was determined by using the end temperature of the phenomena and applying the shape factor.

However, MC evolved into two TAG components so slowly that a period of 17 months was not sufficient for observation of the total disappearance of MC. For example, Fig. S2 (Supplementary Material) illustrates the XRD patterns of 50PPO:50OOP obtained after 11 and 17 months of incubation, and no significant difference can be noted. 
Figure 8 illustrates the phase domains of PPO-OOP mixtures. The eutectic point should be located between the 10PPO:90OOP and 20PPO:80OOP compositions.

$\mathrm{T}\left({ }^{\circ} \mathrm{C}\right)$

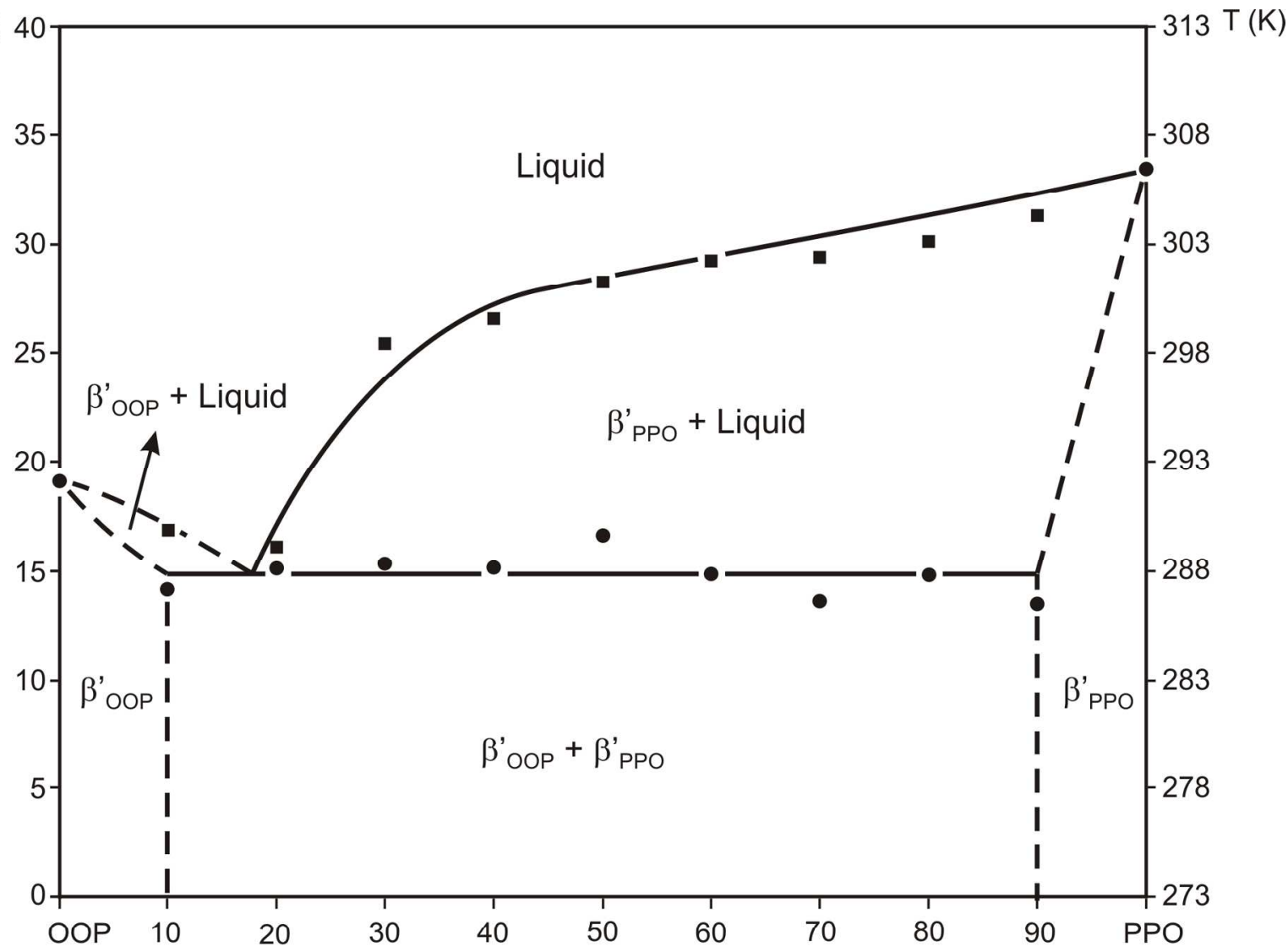

PPO concentration ( $\%$ molar)

Figure 8. Phase behavior of PPO-OOP mixtures, based on DSC onset and end temperatures $\left(\mathrm{T}_{\text {end }}-\Delta \mathrm{T}_{\text {end }}\right)$. Dashed lines represent solubility domain limits, which were defined by extrapolation, not by precise experimental determination. 


\title{
Binary phase behavior of 1,2-dipalmitoyl-3-oleoyl glycerol and 1,3-dioleoyl-2-palmitoyl glycerol (PPO-OPO)
}

\begin{abstract}
Samples were examined by DSC and XRD after 6, 11, and 17 months of incubation, and eutectic behavior of the PPO-OPO binary system was confirmed from the earliest experiments. Here we present only data obtained after 17 months of incubation. Figure 9a plots the DSC thermograms obtained when PPO-OPO mixtures were heated from $0^{\circ} \mathrm{C}$ to $50^{\circ} \mathrm{C}$ at a rate of $2^{\circ} \mathrm{C} \cdot \mathrm{min}^{-1}$. In order to identify the polymorphic forms, laboratory-scale XRD experiments were carried out at $10^{\circ} \mathrm{C}$ for all the compositions (Fig. 9b).

Single endothermic DSC peaks were observed for pure OPO, corresponding to the melting temperature of the $\beta$ form of OPO, and for pure PPO, corresponding to the melting temperature of $\beta$ ' form of PPO. Two endothermic melting peaks were detected for all mixtures from 10PPO:90OPO to 90PPO:10OPO. The temperature of the second peak increased with increasing PPO concentration. In contrast, the first melting peak, corresponding to the OPO fraction, appeared at a constant temperature from the mixture of 10PPO:900PO to 90PPO:10OPO. With increasing PPO concentration, the intensity of the second peak, corresponding to the PPO fraction, increased at the expense of the first melting DSC peak.
\end{abstract}



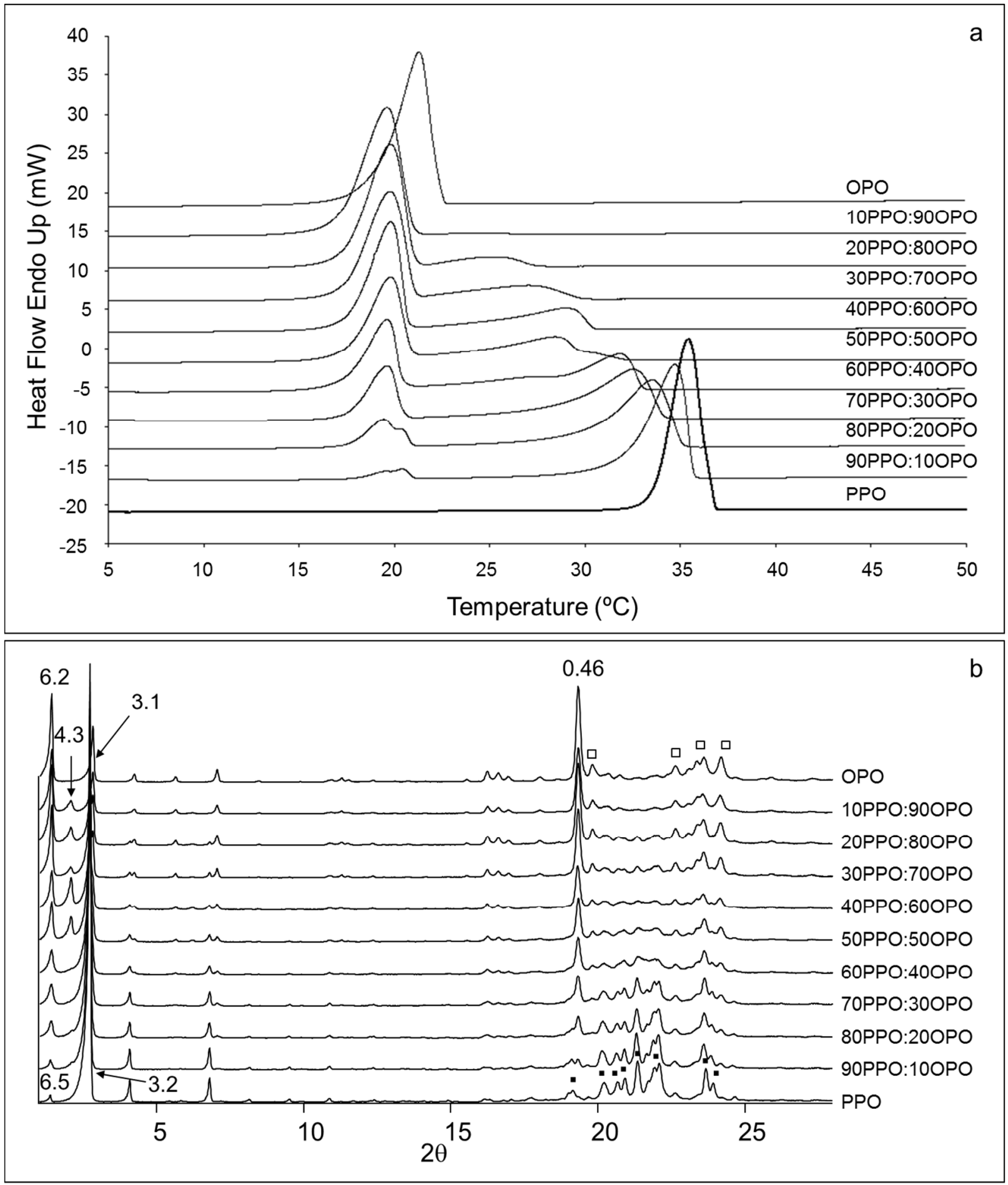

Figure 9. a) DSC heating thermopeaks of PPO-OPO mixtures. b) XRD paterns of PPO-OPO mixtures at $10^{\circ} \mathrm{C}$. Data were obtained after an incubation period of 17 months. 
Polymorphic forms were determined using XRD measurements (Fig. 9b). XRD diffraction peaks in the short and wide-angle region identified single $\beta$ form for pure OPO. Thus, a peak with a long spacing of $6.2 \mathrm{~nm}$ was detected, whereas the typical $\beta$ form peak at $0.46 \mathrm{~nm}$ appeared in the wide-angle region. The d-spacings of other $\beta$ peaks (denoted by $\square$ ) were $0.44 \mathrm{~nm}, 0.39 \mathrm{~nm}$, $0.38 \mathrm{~nm}$, and $0.37 \mathrm{~nm}$. Intensity of the XRD peak with a short spacing of 0.46 progressively decreased as PPO concentration increased. Therefore, typical XRD peaks corresponding to $\beta^{\prime}$ form of PPO became more important. The XRD pattern of pure PPO exhibited a triple-chainlength structure of $\beta$ ' form, with a long spacing of $6.5 \mathrm{~nm}$. The $\beta$ ' form XRD peaks that appeared in the wide-angle region (denoted by *) had short spacings of $0.46 \mathrm{~nm}, 0.44 \mathrm{~nm}, 0.43 \mathrm{~nm}, 0.42 \mathrm{~nm}$, $0.42 \mathrm{~nm}, 0.40 \mathrm{~nm}, 0.38 \mathrm{~nm}$, and $0.37 \mathrm{~nm}$. Mixing the PPO and OPO samples produced no new peaks in the wide-angle region of the XRD patterns at any of the PPO-OPO ratios, aside from those of the PPO and OPO fractions. However, mixtures from 10PPO:90OPO to 50PPO:50OPO (OPO-rich region) exhibited a SAXD peak with a long spacing of 4.3nm. This XRD peak, indicating a double-chain-length structure, may correspond to a metastable $\beta$ ' form of OPO (see Table 1). It should be noted that during the 17 months of stabilization, when the state of the samples was checked after 6 and 11 months of incubation, a decrease of the $4.3 \mathrm{~nm}$ intensity peak was observed for some PPO-OPO mixtures. Thus, this metastable $\beta$, form of OPO tended to disappear while samples stabilized thermodynamically.

Figure 10 depicts the phase behavior of PPO-OPO mixtures constructed at onset and end temperatures in the DSC experiments, taking into account the corresponding shape factor $\Delta \mathrm{T}_{\mathrm{end}}$, as described above. 
$\mathrm{T}\left({ }^{\circ} \mathrm{C}\right)$

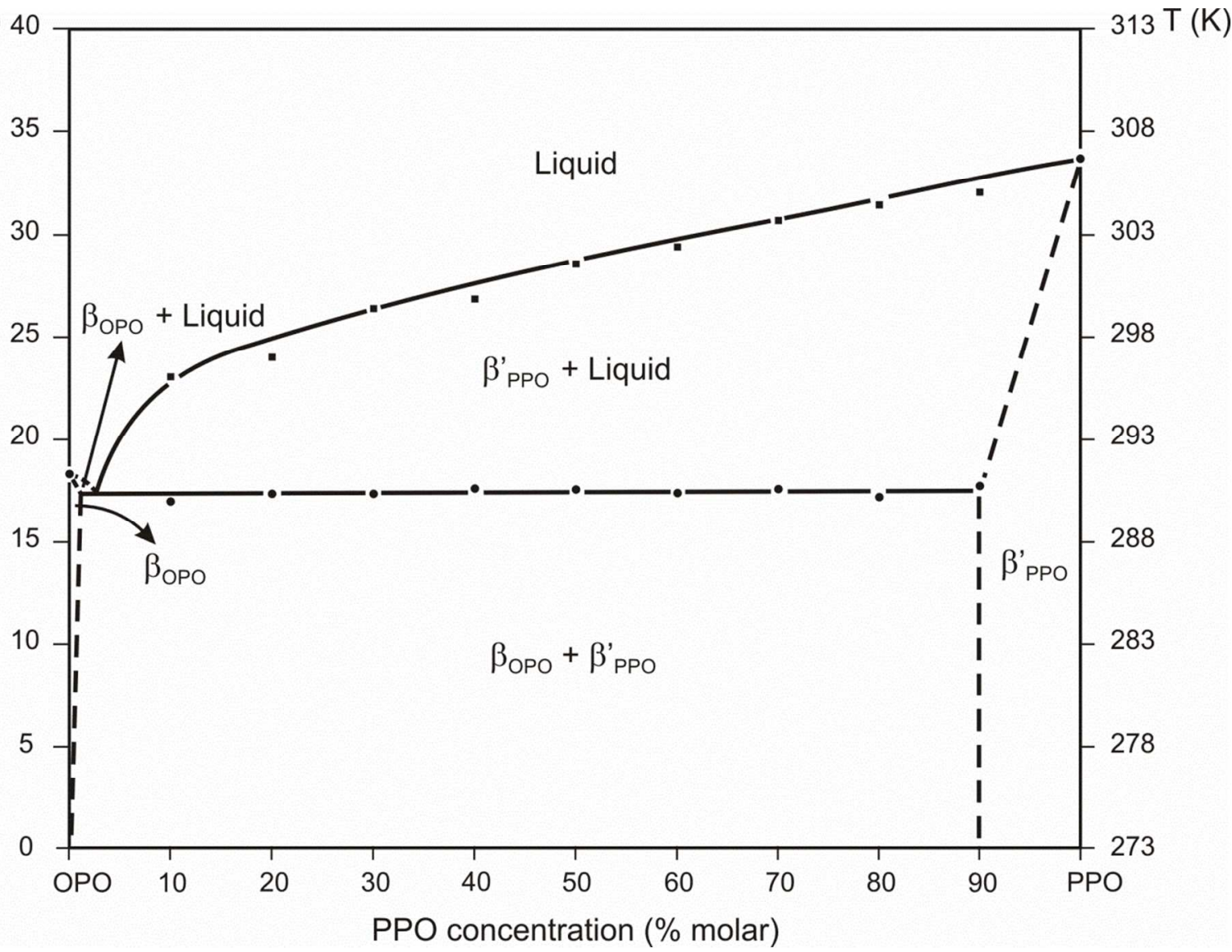

Figure 10. Phase behavior of PPO-OPO mixtures, based on DSC onset and end temperatures $\left(\mathrm{T}_{\text {end }}-\Delta \mathrm{T}_{\text {end }}\right)$. Dashed lines represent solubility domain limits, which were defined by extrapolation, not by precise experimental determination.

PPO and OPO became immiscible, indicating a eutectic property. The exact position of the eutectic point could not be determined, as mixtures were prepared for every $10 \%$. Nevertheless, it was estimated to be located at a composition between pure OPO and 10PPO:90OPO.

\section{DISCUSSION}


Based on the present experiment results, we sought to address two problems: (1) why the MC crystals of OOP-OPO and PPO-OOP were metastable, whereas the MC crystals of POP-OPO and POP-PPO were stable, and (2) why the MC crystals occurred as metastable forms from liquid state.

Regarding the first problem, eutectic behavior was confirmed for the PPO-OPO binary system, whereas OOP-OPO and PPO-OOP were metastable MC-forming. Several polymorphic forms were detected for the two molecular compounds by applying different cooling rates to the 50:50 compositions and by incubating the binary mixtures for 17 months. Table 2 lists the long and short spacings of the polymorphic forms of $\mathrm{MC}_{\mathrm{OOP}: \mathrm{OPO}}$ and $\mathrm{MC}_{\mathrm{PPO}: \mathrm{OOP}}$.

Table 2. Long spacing (LS, $\mathrm{nm}$ ) and short spacing (SS, nm) values of polymorphic forms of OOP:OPO and PPO:OOP molecular compounds.

\begin{tabular}{lllllll}
\hline & \multicolumn{7}{c}{ MC $_{\text {OOP:OPO }}$} & \multicolumn{5}{c}{ MC $_{\text {PPO:OOP }}$} \\
\hline Polymorph & sub- $\alpha_{\text {OOP:OPO }}$ & $\beta^{\prime}{ }_{\text {OOP:OPO }}$ & $\beta_{\text {OOP:OPO }}$ & sub- $\alpha_{\text {PPO:OOP }}$ & $\alpha_{\text {PPO: OOP }}$ & $\beta_{\text {PPO:OOP }}$ \\
\hline LS (nm) & 5.6 & 4.4 & 4.2 & 4.9 & 4.9 & 4.2 \\
\hline SS (nm) & 0.41 & 0.45 & 0.46 & 0.42 & 0.41 & 0.42 \\
& 0.39 & 0.43 & nd. & 0.38 & & 0.39 \\
& & 0.42 & & & & \\
& & 0.39 & & & & \\
\hline
\end{tabular}

nd. Not determined

However, after thermodynamic stabilization at a constant temperature, we confirmed that both molecular compounds tended to evolve into the corresponding TAG components, indicating 
eutectic property. It may take a long time, though, to reach thermodynamically stable eutectic phase systems. We did not confirm the complete separation of MC into two TAG component molecules after 17 months of incubation, as MC and TAG components still coexisted. Such phase behavior was not detected in the mixtures of POP-OPO ${ }^{20}$ and POP-PPO ${ }^{19}$, in which the $\beta$ form of the molecular compound was stable and no evolution occurred in the two mixture systems.

The fact that PPO-OPO mixtures were immiscible, not forming a molecular compound, and that OOP-OPO and PPO-OOP mixtures were MC-forming (although in both cases they seemed to be metastable) can be explained by taking into account the molecular interactions between the molecular TAG components.

The main molecular interactions that influence stabilization of crystal structures of TAGs containing saturated and unsaturated fatty acids are ${ }^{1}$ :

(i) Aliphatic chain packing, formed by molecular interactions between saturated and unsaturated fatty-acid chains.

(ii) Glycerol conformation, which determines the configuration of all TAG molecules.

(iii) Methyl end stacking, which determines the chain inclination and the chain-length structure.

The ability or inability to form a molecular compound having a double-chain-length structure may be clarified by considering the combined effects of these interactions.

Figure 11a illustrates different glycerol conformations of two asymmetric units of TAG molecules in their most stable form. 


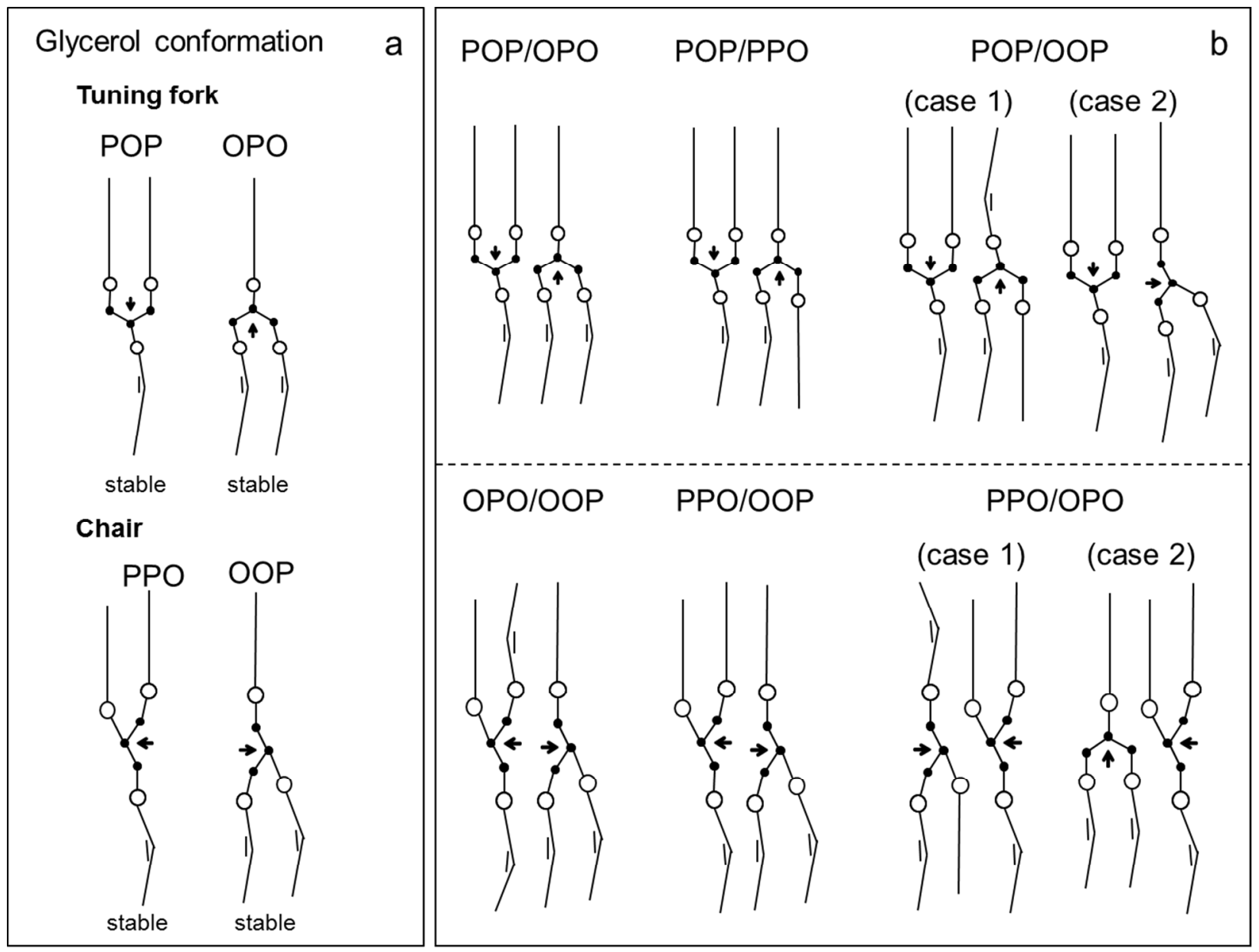

Figure 11. a) Structure models of TAGs containing palmitic and oleic fatty acids (tuning fork and chair conformation). b) Structure models of molecular compounds of TAGs containing palmitic and oleic fatty acids. POP-OPO, POP-PPO and POP-POO cases were reported by Minato et al. ${ }^{20,19}$ and Zhang et al. ${ }^{18}$, respectively.

Glycerol conformation is symbolized using the direction of the glycerol group, indicated by an arrow pointing at the sn-2 carbon atom, and defines the direction between the middle point of the two glycerol carbon atoms at the first and third positions, and the glycerol carbon atom at the second position. The glycerol conformation determines the lateral chain packing of palmitic and 
oleic acid moieties. In more stable conformations, the palmitic and oleic acid chains should be packed in separated leaflets. In contrast, the coexistence of palmitic and oleic acid in the same leaflet may decrease the packing coefficient ${ }^{15}$ in aliphatic chain packing. Thus, the conformation obtained should become metastable. As Craven et al. ${ }^{14}$ reviewed, typically, chiral TAG adopt the chair conformation while achiral TAG adopt the tuning fork. Therefore, the most stable conformation for symmetric (achiral) POP and OPO is tuning fork, ${ }^{32}$ and asymmetric (chiral) PPO and OOP may adopt a chair conformation.

Figure $11 \mathrm{~b}$ depicts possible structure models of molecular compounds of TAGs containing palmitic and oleic fatty acids. The three structure models in the upper part had previously been reported, ${ }^{18-20}$ whereas the three models in the bottom are proposed in the present work.

The neighboring glycerol groups in parallel arrangement are directed along the chain axis with an opposite turn in the POP-OPO mixture. ${ }^{20}$ The tuning fork conformation is the most stable for both POP and OPO (Fig. 11b), and it enables the oleoyl and palmitoyl chains to make separate leaflets with stabilized aliphatic interactions. Hence, $\mathrm{MC}_{\mathrm{POP}: \mathrm{OPO}}$ is stable.

For POP:PPO molecular compound formation, we assume that both TAGs may adopt the tuning fork conformation depicted in Fig. 11b. With this structure, the adjacent glycerol groups should be directed along the chain axis with an opposite turn, similar to those in the POP-OPO mixture. However, palmitoyl and oleoyl chains are inevitably located in one leaflet, and the other leaflet may be fully occupied by palmitoyl chains. ${ }^{19}$ Chain packing of the palmitoyl-palmitoyl leaflet may be stabilized, but steric hindrance may occur; however, this hindrance does not destabilize the total MC molecule. Thus, the glycerol group interactions and palmitoyl-palmitoyl interactions may prevail against destabilization in the chain packing of the palmitoyl-oleoyl leaflet. 
Previous study indicated that POP-OOP exhibited immiscible eutectic behavior, with no molecular compound formation, and two possible structure models were investigated to clarify this result (Fig. 11b). ${ }^{18}$ The model of case 1 assumes a parallel direction for the glycerol groups, which may be more stabilized than the unparallel direction in case 2. However, the structural model of case 1 may destabilize the acyl chain packing because of the coexistence of oleoyl and palmitoyl chains in the two leaflets. In case 2, the oleoyl and palmitoyl chains are separately placed in their own leaflets. Consequently, neither of the two possible models of the POP:OOP molecular compound may alleviate destabilization of the acyl chain packing, and no molecular compound was observed in the POP-OOP mixture.

In the present work, as expected, eutectic behavior was also observed in the PPO-OPO binary mixtures, and no molecular compound formed. Figure $11 \mathrm{~b}$ presents two possible models of PPO:OPO molecular compound. In case 1, the two TAGs adapt a chair conformation, and the adjacent glycerol groups are parallel to each other and perpendicularly directed to the chain axis, and thus parallel to the lamellar plane. However, this structure may be destabilized due to chain packing of the two leaflets, where palmitoyl-oleoyl steric hindrance occurs. In contrast, in case 2, no steric hindrance takes place in the aliphatic chain packing, as oleoyl and palmitoyl chains are located in different leaflets. However, this structure may be destabilized because of steric hindrance between tuning fork (OPO) and chair (PPO) conformations, as adjacent glycerol groups are perpendicular to each other.

We observed formation of the molecular compound having a double-chain-length structure in the OOP-OPO system. However, thermal stabilization experiments indicated that this molecular compound was metastable and tended to separate into two TAG components, so that the system became eutectic. In the proposed structure model depicted in Fig. 11b, the directions of glycerol 
groups are parallel to the lamellar plane, and OOP and OPO have chair-type glycerol conformations. Chain packing of the oleoyl-oleoyl leaflet may also be stabilized, yet the packing coefficient for the palmitoyl-oleoyl leaflet may decrease. Although this fact did not destabilize the total MC structure in its metastable state, long-term incubation caused the binary mixtures to evolve into a eutectic system. Taking into account our proposed model, the glycerol conformation of $\mathrm{OPO}^{14}$ was probably converted from less stable chair-type to more stable tuning fork-type during this stabilization, causing higher steric hindrance in the palmitoyl-oleoyl leaflet.

A metastable molecular compound also formed in the PPO-OOP binary mixtures. The proposed structure model of the PPO:OOP molecular compound is depicted in Fig. 11b, with the chair conformation of the glycerol groups and separate chain packing of palmitoyl and oleoyl chains, which may increase the packing coefficient. However, experiment results indicated metastability of this molecular compound. A possible cause of the metastability of this MC structure is the racemicity of PPO and POO. MC-forming mixtures may be destabilized to form eutectic mixtures of R-PPO:S-PPO ${ }^{33}$ and R-OOP:S-OOP. Further studies are needed to determine the mixing behavior of the enantiopure TAGs $(\mathrm{R} / \mathrm{S})$.

Thus, repulsive interactions between glycerol groups and fatty acid moieties of OOP-OPO and PPO-OOP may cause separation from MC to eutectic separated phases after a long incubation period. The 17 months at the incubation temperatures examined in this study may not be sufficient to completely separate MC into two TAG components.

Regarding the second problem with metastable molecular compounds for OOP-OPO and PPOOOP, we consider the molecular structures of neat liquid of TAGs as a hypothesis. Larsson ${ }^{34}$ first proposed ordering in liquid state, advocating the existence of liquid-crystal-like lamellae. 
According to Larsson's paralamellar model, lamellar liquid crystals with short-range order exist in TAG melts, and this lamellar ordering of some TAGs in the liquid state can persist at temperatures up to $40^{\circ} \mathrm{C}$ above the melting point of the highest melting polymorph. In contrast, Cebula et al. ${ }^{35}$ argued against Larsson's lamellar model in favor of a nematic-like structure. Later, Corkery et al. ${ }^{36}$ proposed a Discotic Model for molten TAGs, in which TAG molecules in melt form Y-shaped conformers that are loosely bound individually within disk-like volumes and can self-assemble into discotic mesophases.

The above three models describe some ordering in the liquid state of TAGs. Thus, for the first stage of MC formation, we assume that as a precursor, molecular aggregation having some ordering (i.e., lamellar structure) may exist. MC with a double-chain-length structure may occur as a metastable phase from the liquid state, in accordance with the Ostwald step rule. ${ }^{37} \mathrm{MC}$ crystals form as stable structures for POP-OPO and POP-PPO, and tend to separate during incubation into two TAG components (more stable) for OOP-OPO and PPO-OOP, due to repulsive interactions between glycerol groups and fatty acid chains.

\section{CONCLUSIONS}

We determined the phase behavior of PPO-OPO, OOP-OPO and PPO-OOP binary mixtures. The PPO-OPO system exhibited eutectic behavior, whereas OOP-OPO and PPO-OOP were MCforming in the metastable state. Long incubation periods were needed to determine the evolution of the binary mixtures, and the results demonstrated that $\mathrm{MC}_{\mathrm{OOP}: \mathrm{OPO}}$ and $\mathrm{MC}_{\mathrm{PPO}: \mathrm{OOP}}$ were metastable and tended to separate into pure TAG components. It was shown that thermodynamic 
equilibrium is highly difficult to reach, in which time and temperature of incubation become key factors.

Non-formation of molecular compounds in PPO-OPO and metastability of $\mathrm{MC}_{\mathrm{OOP}: \mathrm{OPO}}$ and $\mathrm{MC}_{\mathrm{PPO}: \mathrm{OOP}}$ were investigated, taking into account the main molecular interactions that influence stabilization of crystal structures of TAGs containing saturated and unsaturated fatty acids. These interactions consist mainly of aliphatic chain packing, glycerol conformation of the TAG components, and methyl end stacking. The importance of stereochemistry of asymmetric TAGs for understanding their mixing behavior was again evidenced. 


\section{ASSOCIATED CONTENT}

Supporting Information. Figures showing XRD patterns observed after 11 and 17 months of incubation of 50OOP:50OPO (Figure S.1.) and 50PPO:50OOP (Figure S.2.). This material is available free of charge via the Internet at http://pubs.acs.org.

\section{AUTHOR INFORMATION \\ Corresponding Author \\ Phone: +34 9340213 50. Fax: +34 9340213 40. E-mail: laurabayes@ub.edu}

\section{ACKNOWLEDGMENT}

The authors acknowledge the financial support of the Ministerio de Economía $y$ Competitividad through Project MAT2011-27225, the Generalitat de Catalunya through the Grup Consolidat (SGR 2009 1307), and the Ministerio de Educación y Ciencia through the Factoría Cristalográfica (Consolider-Ingenio CSD2006-15) and through the Beca del Programa de Formación de Profesorado Universitario (FPU). SR-XRD experiments were performed with the approval of the Photon Factory Program Advisory Committee (proposal no. 2012G166). The authors gratefully acknowledge the help of Associate Prof. N. Igarashi and N. Shimizu, Station Manager of Beamline 6A at Photon Factory (KEK Institute, Tsukuba, Japan). 


\section{REFERENCES}

1. Lipids: Structure, Physical Properties and Functionality; Larsson, K.; Quinn, P.; Sato, K., Tiberg, F., Eds.; The Oily Press: Bridgwater, 2006.

2. Structure and Properties of Fat Crystal Networks; Marangoni, A.G.; Wesdrop, L. H., Eds.; CRC Press: Boca Raton, 2013.

3. Sato, K.; Ueno, S. in Fats in Food Technology; Rajah, K. S., Ed.; Wiley Blackwell: Chichester, 2014, 1-38.

4. Physical Properties of Lipids; Marangoni, A. G.; Narine, S. S., Eds.; Marcel Dekker: New York, USA, 2002, 63-217.

5. Sato, K.; Bayés-García, L.; Calvet, T.; Cuevas-Diarte, M. A.; Ueno, S. External factors affecting polymorphic crystallization of lipids. Eur. J. Lipid Sci. Technol. 2013, 115, 12241238.

6. Talbot, G. in Cocoa Butter and Related Compounds; Garti, N.; Widlak, N. R., Eds.; AOCS Press: Urbana, 2012, 1-33.

7. Timms, R. E. Fractional crystallization-the fat modification process for the $21^{\text {st }}$ century. Eur. J. Lipid Sci. Technol. 2005, 107, 48-57. 
8. Jiménez Márquez, A.; Beltrán Maza, G.; Aguilera Herrera, M. P.; Uceda Ojeda, M. Differential scanning calorimetry. Influence of virgin olive oil composition on its termal profile. Grasas Aceites 2007, 58, 122-129.

9. Díaz, I.; García Regueiro, J. A.; Casillas, M.; De Pedro, E. Triglyceride composition of fresh ham fat from Iberian pigs produced with different systems of animal nutrition. Food Chem. 1996, 55, 383-387.

10. Petrón, M. J.; Muriel, E.; Timón, M. L.; Martín, L.; Antequera, T. Fatty acids and triacylglycerols profiles from different types of Iberian dry-cured hams. Meat Sci. 2004, $68,71-77$.

11. Kodali, D. R.; Atkinson, D.; Small, D. M. Polymorphic behavior of 1,2-dipalmitoyl-3lauroyl(PP12)- and 3-myristoyl(PP14)-sn-glycerols. J. Lipid Res. 1990, 31, 1853-1864.

12. Boodhoo, M. V.; Bouzidi, L.; Narine, S. S. The binary phase behavior of 1,3-dicaproyl-2stearoyl-sn-glycerol and 1,2-dicaproyl-3-stearoyl-sn-glycerol. Chem. Phys. Lipids 2009, $157,21-39$.

13. Craven, R. J.; Lencki, R. W. Crystallization, Polymorphism, and Binary Phase Behavior of Model Enantiopure and Racemic Triacylglycerols. Cryst. Growth Des. 2011, 11, 17231732.

14. Craven, R. J.; Lencki, R. W. Polymorphism of Acylglycerols: A Stereochemical Perspective. Chem. Rev. 2013, 113, 7402-7420.

15. Kitaigorodsky, A. I. Mixed Crystals; Springer Series in Solid-State Sciences, SpringerVerlag: Berlin Heidelberg, 1984, vol. 33. 
16. Sato, K.; Ueno, S. in Bailey’s Industrial Oil and Fat Products; Shahidi, F., Ed.; John Wiley \& Sons Inc.: Hoboken, 2005, vol. 1, 77-120.

17. Sato, K.; Ueno, S. in Crystallization Processes in Fats and Lipid Systems; Garti, N.; Sato, K., Eds.; Marcel Dekker: New York, 2001, 177-209.

18. Zhang, L.; Ueno, S.; Miura, S.; Sato, K. Binary Phase Behavior of 1,3-Dipalmitoyl-2oleoyl-sn-glycerol and 1,2-Dioleoyl-3-palmitoyl-rac-glycerol. J. Amer. Oil Chem. Soc. 2007, 84, 219-227.

19. Minato, A.; Ueno, S.; Smith, K.; Amemiya, Y.; Sato, K. Thermodynamic and Kinetic Study on Phase Behavior of Binary Mixtures of POP and PPO Forming Molecular Compound Systems. J. Phys. Chem. B 1997, 101, 3498-3505.

20. Minato, A.; Ueno, S.; Yano, J.; Smith, K.; Seto, H.; Amemiya, Y.; Sato, K. Thermal and Structural Properties of sn-1,3-Dioleoyl-2-palmitoylglycerol Binary Mixtures Examined with Synchrotron Radiation X-Ray Diffraction. J. Am. Oil Chem. Soc. 1997, 74, 12131220.

21. Ikeda, E.; Ueno, S.; Miyamoto, R.; Sato, K. Phase Behavior of a Binary Mixture of 1,3Dipalmitoyl-2-oleoyl-sn-glycerol and 1,3-Dioleoyl-2-palmitoyl-sn-glycerol in n-Dodecane Solution. J. Phys. Chem. B 2010, 114, 10961-10969.

22. Zhang, L.; Ueno, S.; Sato, K.; Adlof, R. O.; List, G. R. Thermal and structural properties of binary mixtures of 1,3-distearoyl-2-oleoyl-glycerol (SOS) and 1,2-dioleoyl-3-stearoyl-snglycerol (sn-OOS). J. Therm. Anal. Calorim., 2009, 98, 105-111. 
23. Engström, L. Triglyceride Systems Forming Molecular Compounds. J. Fat Sci. Technol. 1992, 94, 173-181.

24. Koyano, T.; Hachiya, I.; Sato, K. Phase Behavior of Mixed Systems of SOS and OSO. J. Phys. Chem. 1992, 96, 10514-10520.

25. Takeuchi, M.; Ueno, S.; Yano, J.; Floter, E.; Sato, K. Polymorphic transformation of 1,3distearoyl-sn-2-linoleoyl-glycerol. J. Am. Oil Chem. Soc. 2000, 77, 1243-1249.

26. Bayés-García, L.; Calvet, T.; Cuevas-Diarte, M. A.; Ueno, S.; Sato, K. In situ observation of transformation pathways of polymorphic forms of 1,3-dipalmitoyl-2-oleoyl glycerol (POP) examined with synchrotron radiation X-ray diffraction and DSC. CrystEngComm. 2013, 15, 302-314.

27. Bayés-García, L.; Calvet, T.; Cuevas-Diarte, M. A.; Ueno, S.; Sato, K. In situ synchrotron radiation X-ray diffraction study of crystallization kinetics of polymorphs of 1,3-dioleoyl2-palmitoyl glycerol (OPO). CrystEngComm. 2011, 13, 3592-3599.

28. Miura, S.; Konishi, H. Crystallization behavior of 1,3-dipalmitoyl-2-oleoyl-glycerol and 1palmitoyl-2,3-dioleoyl-glycerol. Eur. J. Lipid Sci. Technol. 2001, 103, 804-809.

29. Bayés-García, L. PhD thesis: Polymorphism and Solid State Miscibility of Triacylglycerols. Application to Food Authentication. University of Barcelona, Barcelona, 2013.

30. Perkin Elmer. Instructions Model DSC-4. Norwalk, Connecticut, USA, 1982. 
31. Corchinoux, R.; Chanh, N. B.; Haget, Y.; Calvet, T.; Estop, E.; Cuevas-Diarte, M. A. Du signal aux phénomènes: une approche practique pour l'établissement des diagrammes de phases par analyse thermique. J. Chim. Phys. 1989, 86, 561-593.

32. Van Mechelen, J. B.; Peschar, R.; Schenk, H. Structures of mono-unsaturated triacylglycerols. I. The $\beta_{1}$ polimorph. Acta Cryst. B 2006, B62, 1121-1130.

33. Mizobe, H.; Tanaka, T.; Hatakeyama, N:; Nagai, T.; Ichioka, K.; Hondoh, H.; Ueno, S.; Sato, K. Structures and Binary Mixing Characteristics of Enantiomers of 1-Oleoyl-2,3dipalmitoyl-sn-glycerol (S-OPP) and 1,2-Dipalmitoyl-3-oleoyl-sn-glycerol (R-PPO). J. Am. Oil Chem. Soc. 2013, 90, 1809-1817.

34. Larsson, K. On the structure of the liquid state of triglycerides. J. Am. Oil Chem. Soc. 1992, 69, 835-836.

35. Cebula, D. J.; McClements, D. J.; Povey, M. J. W.; Smith, P. R. Neutron diffraction studies of liquid and crystalline trilaurin. J. Am. Oil Chem. Soc. 1992, 69, 130-136.

36. Corkery, R. W.; Rousseau, D.; Smith, P.; Pink, D. A.; Hanna, C. B. A Case for Discotic Liquid Crystals in Molten Triglycerides. Langmuir 2007, 23, 7241-7246.

37. Ostwald, W. Studien über die Bildung und Umwandlung fester Körper. Z. Phys. Chem. 1897, 22, 289-330. 


\section{TABLE OF CONTENTS IMAGE}

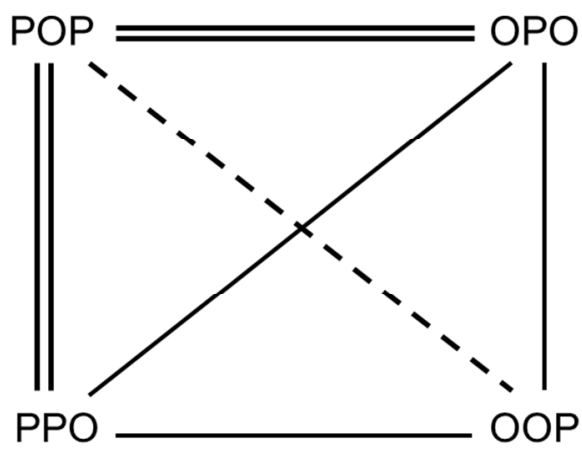

Molecular Compound (metastable and stable states)

- - - - - Eutectic (metastable and stable states)

Present study 


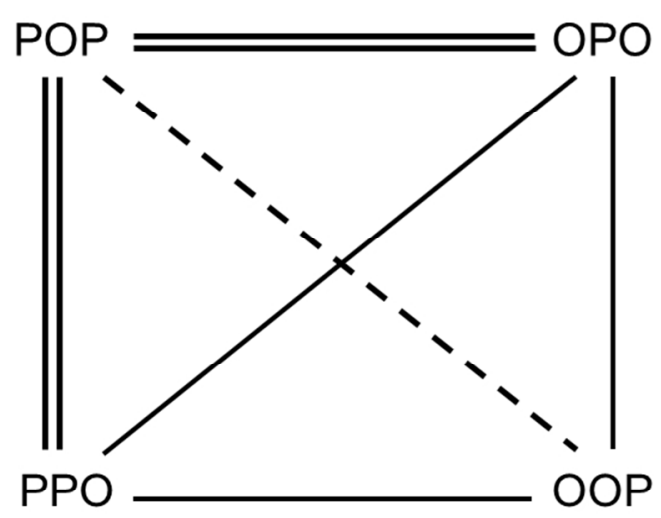

Molecular Compound (metastable and stable states) (19,20 $^{1}$

- - - - - Eutectic (metastable and stable states) $)^{18}$

Present study

Tetragonal diagram of binary mixture systems of POP, OPO, PPO and POO. $184 \times 124 \mathrm{~mm}(150 \times 150 \mathrm{DPI})$ 


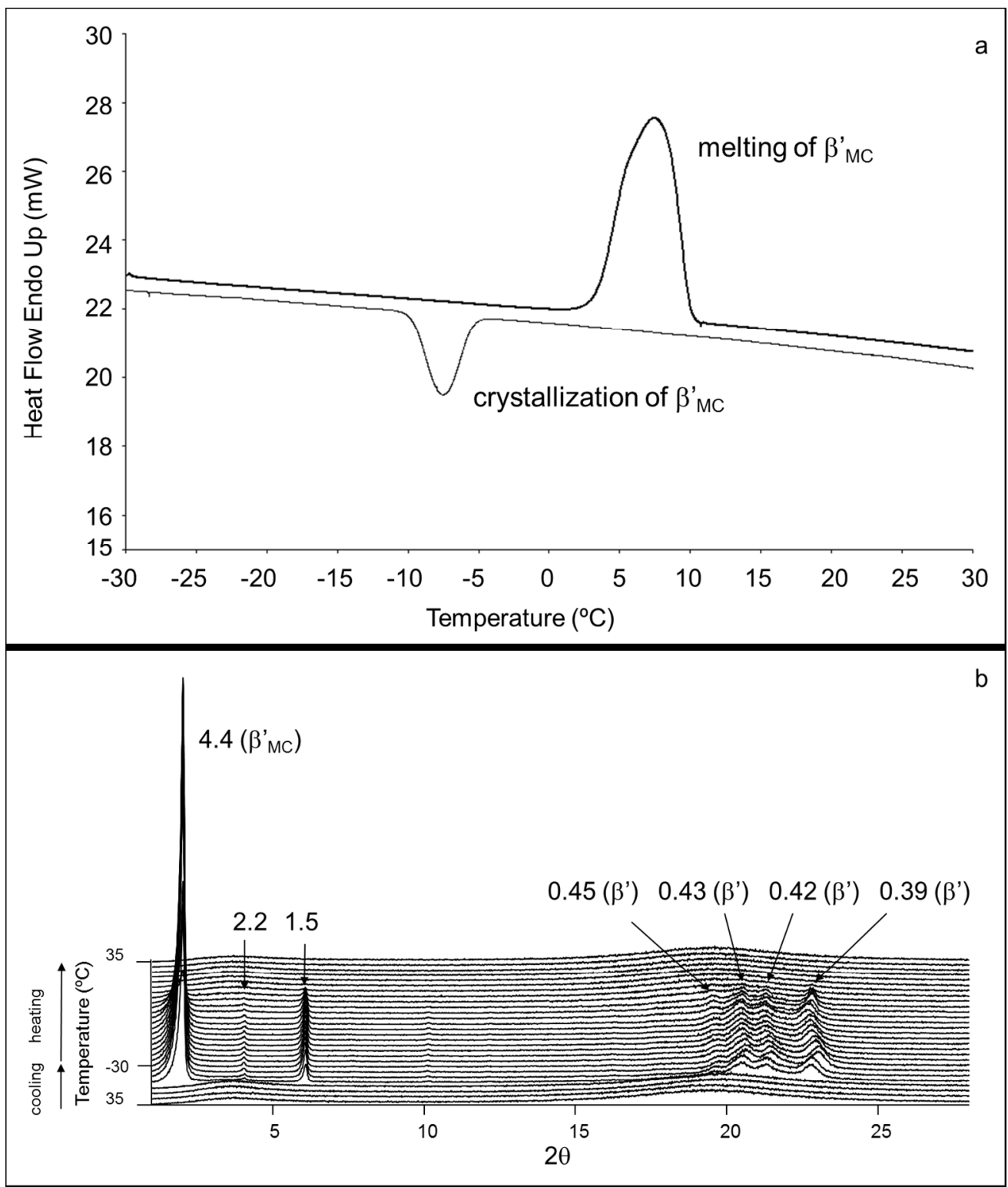

Polymorphic behavior of metastable forms of 5000P:500PO when cooled at $0.5^{\circ} \mathrm{C} \cdot \mathrm{min}^{-1}$ and heated at $2^{\circ} \mathrm{C} \cdot \mathrm{min}^{-1}$. a) DSC thermogram. b) XRD paterns. $258 \times 304 \mathrm{~mm}(150 \times 150 \mathrm{DPI})$ 


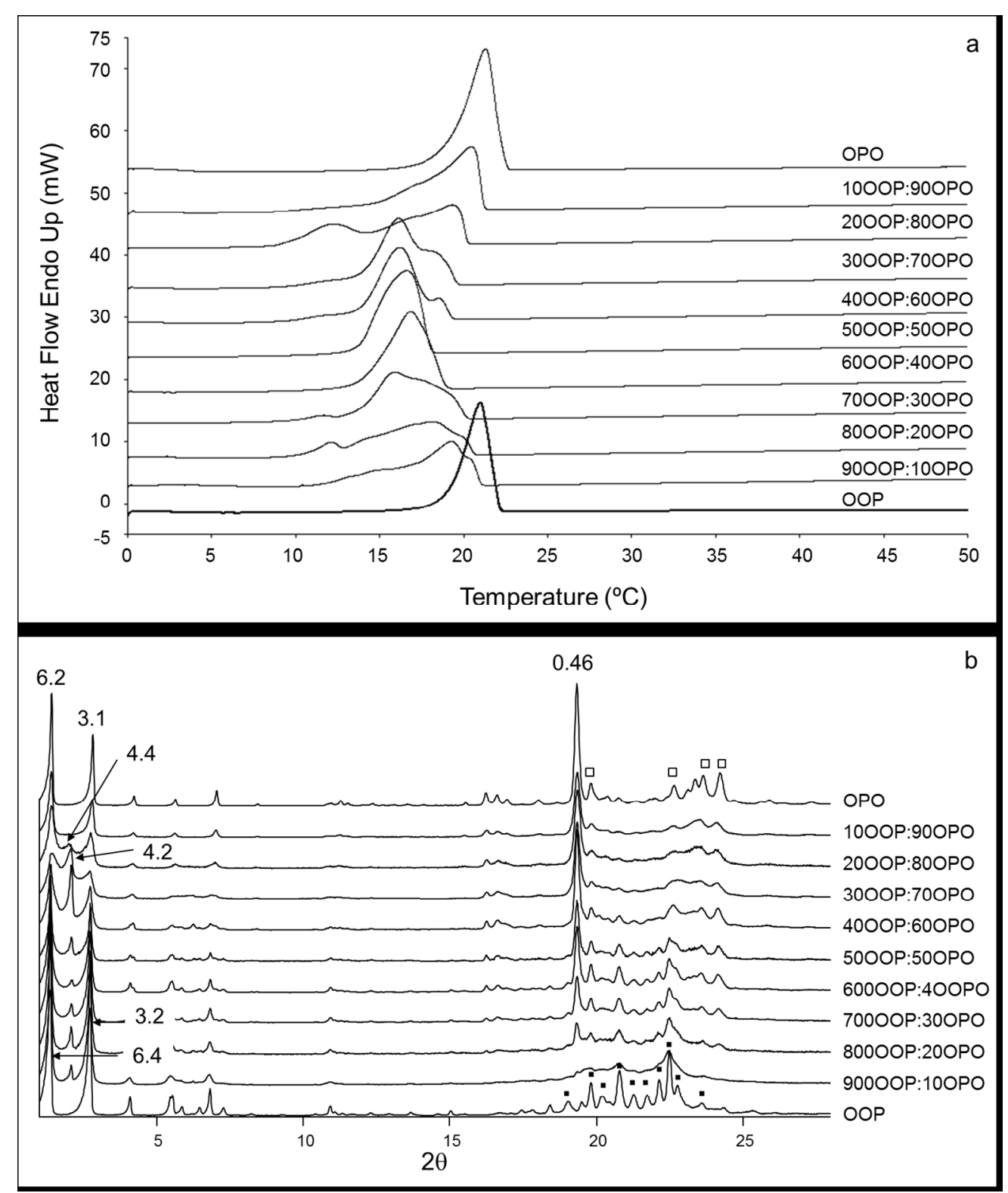

a) DSC heating thermopeaks of OOP-OPO mixtures. b) XRD paterns of OOP-OPO mixtures at $5^{\circ} \mathrm{C}$. Data were obtained after an incubation period of 17 months. $255 \times 305 \mathrm{~mm}(150 \times 150 \mathrm{DPI})$ 


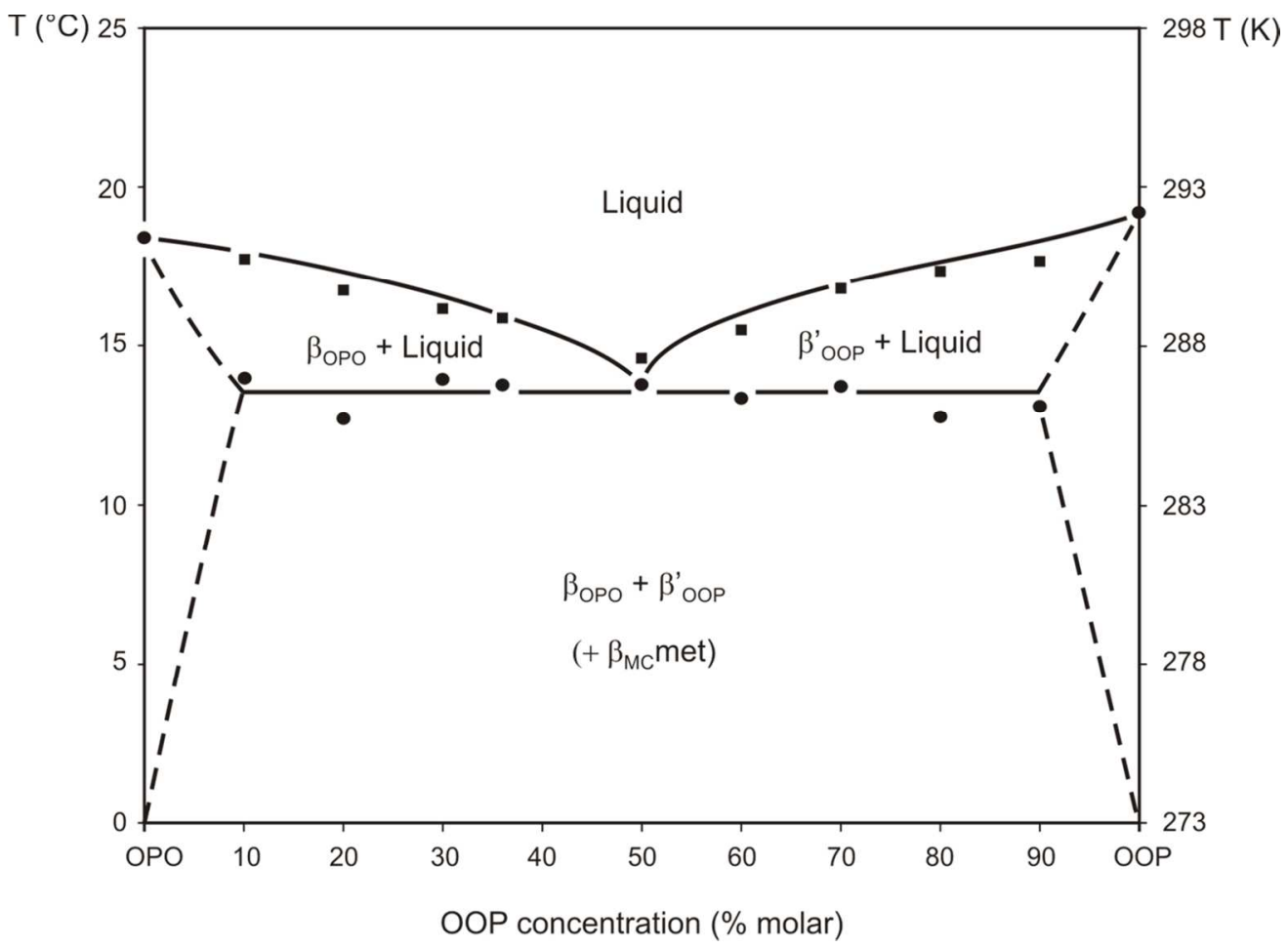

Phase behavior of OOP-OPO mixtures, based on DSC onset and end temperatures ( $\left.T_{\text {end }}-\Delta T_{\text {end }}\right)$. Dashed lines represent solubility domain limits, which were defined by extrapolation, not by precise experimental determination.

$232 \times 169 \mathrm{~mm}(150 \times 150 \mathrm{DPI})$ 
DSC cooling and heating thermopeaks of metastable forms of 50PPO:5000P mixture obtained during cooling and heating processes at a rate of $2^{\circ} \mathrm{C} \cdot \mathrm{min}^{-1}$. $262 \times 165 \mathrm{~mm}(150 \times 150 \mathrm{DPI})$ 


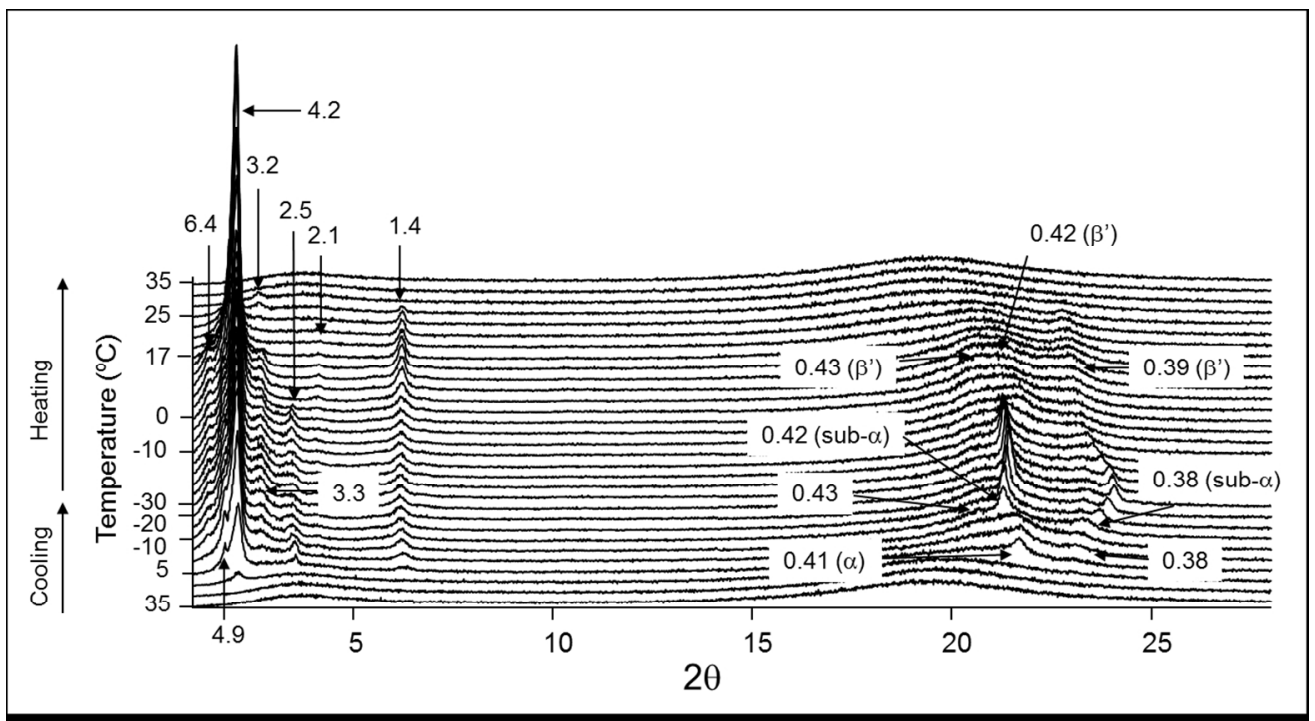

XRD paterns of metastable forms of 50PPO:5000P mixture obtained during cooling and heating processes at a rate of $2^{\circ} \mathrm{C} \cdot \mathrm{min}^{-1}$. $232 \times 127 \mathrm{~mm}(150 \times 150 \mathrm{DPI})$ 

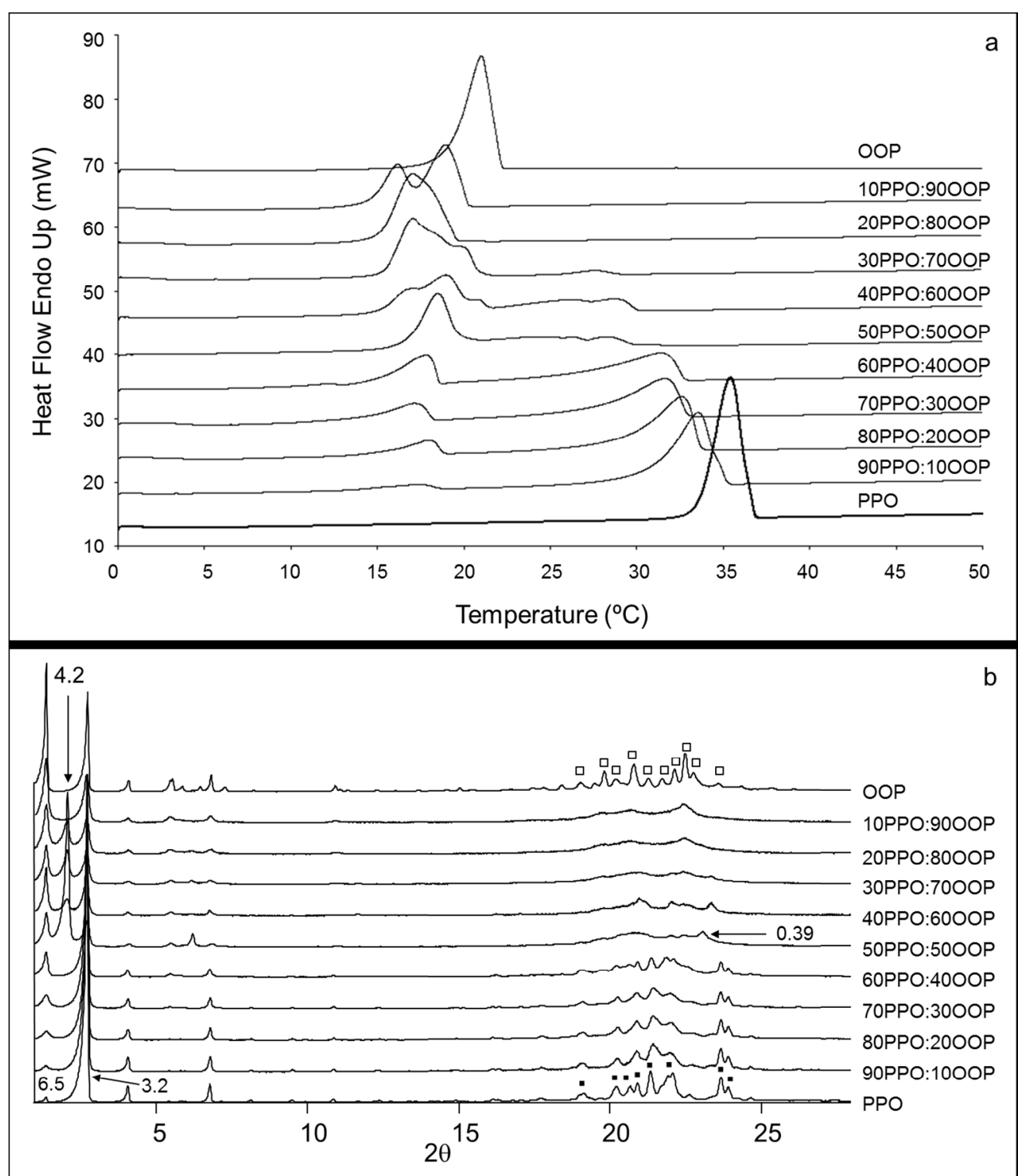

a) DSC heating thermopeaks of PPO-OOP mixtures. b) XRD paterns of PPO-OOP mixtures at $5^{\circ} \mathrm{C}$. Data were obtained after an incubation period of 17 months. $254 \times 294 \mathrm{~mm}(150 \times 150 \mathrm{DPI})$ 


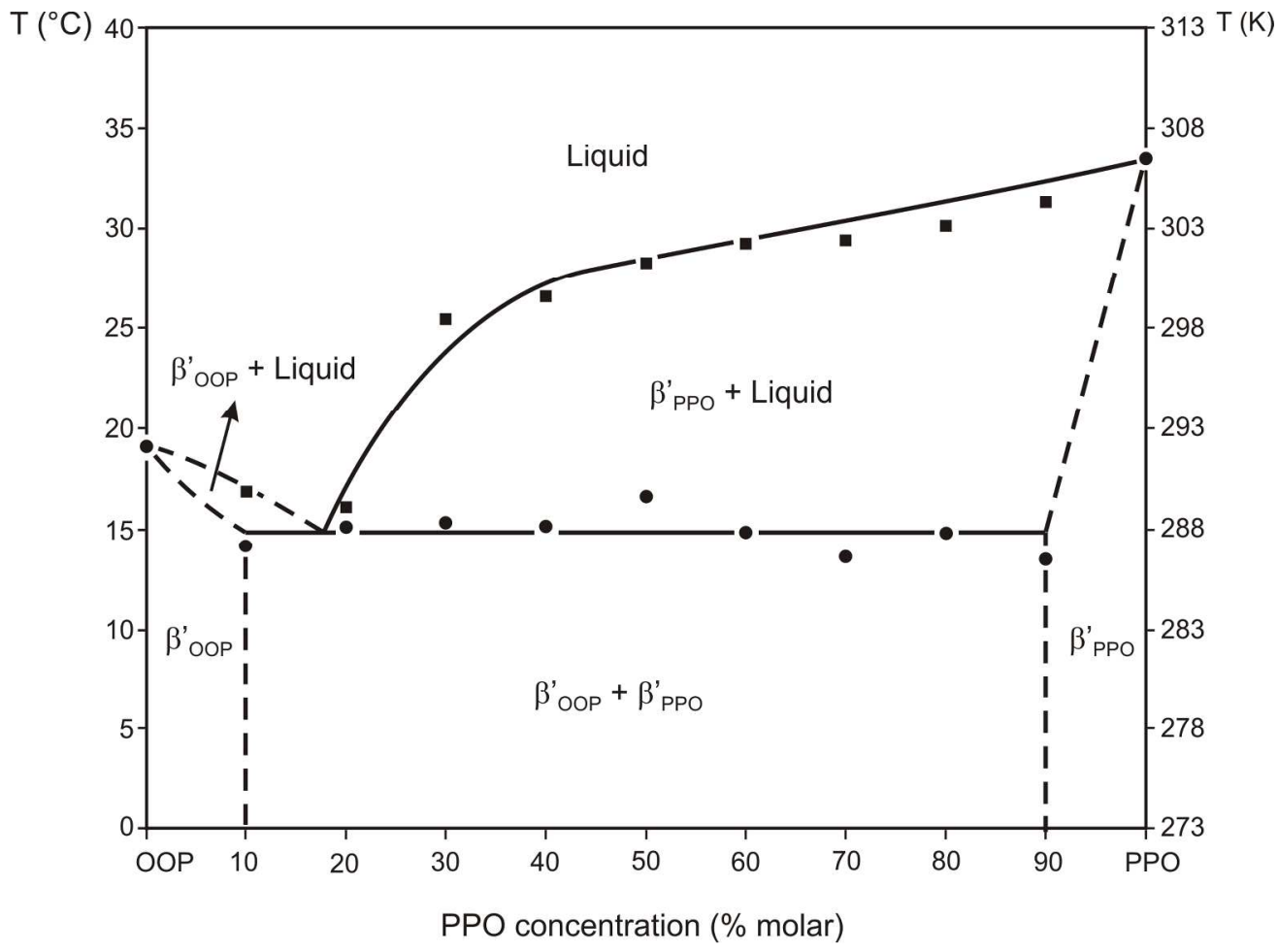

Phase behavior of PPO-OOP mixtures, based on DSC onset and end temperatures ( $T_{\text {end }}-\Delta T_{\text {end }}$ ). Dashed lines represent solubility domain limits, which were defined by extrapolation, not by precise experimental determination. 

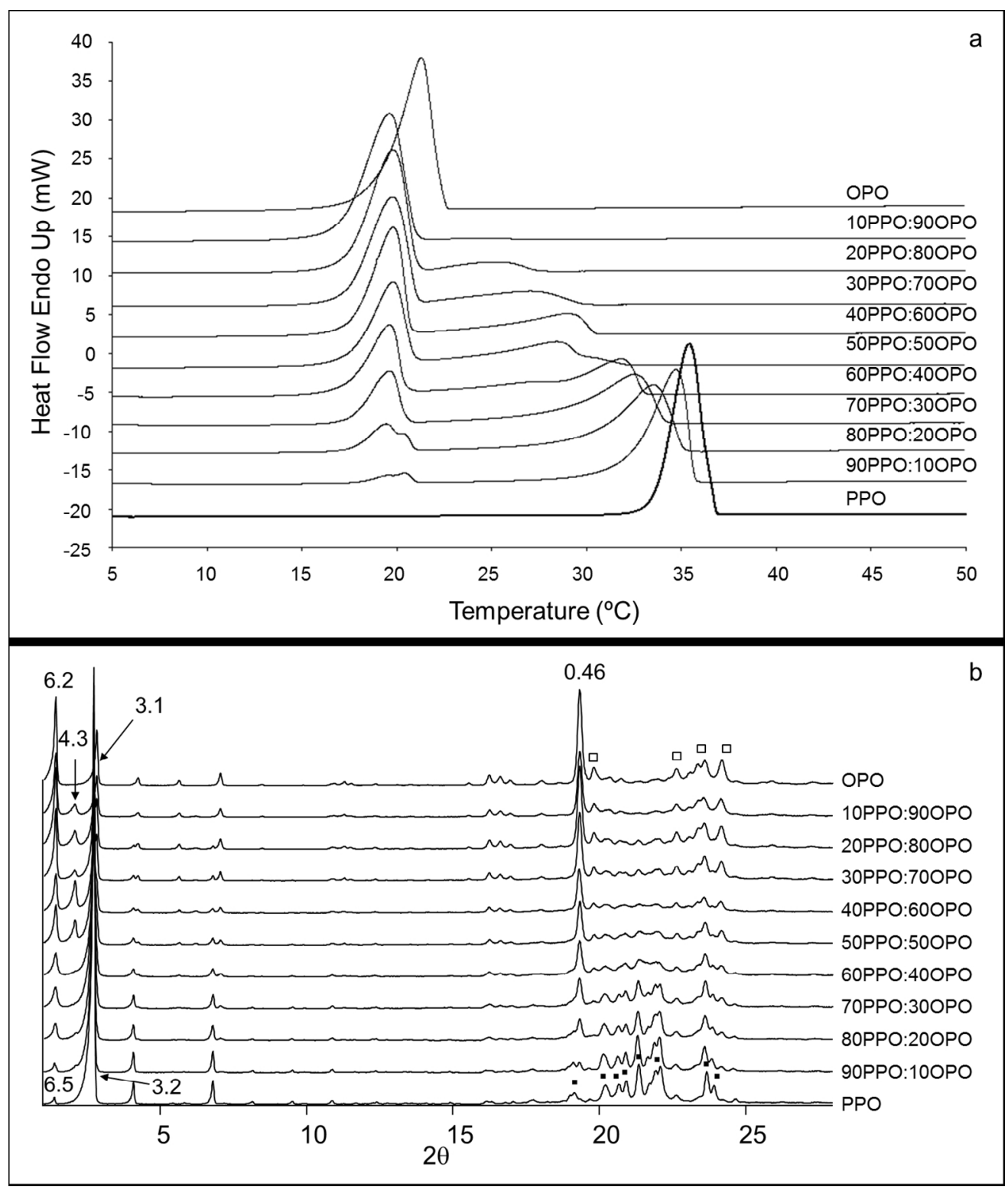

a) DSC heating thermopeaks of PPO-OPO mixtures. b) XRD paterns of PPO-OPO mixtures at $10^{\circ} \mathrm{C}$. Data were obtained after an incubation period of 17 months. $254 \times 299 \mathrm{~mm}(150 \times 150 \mathrm{DPI})$ 


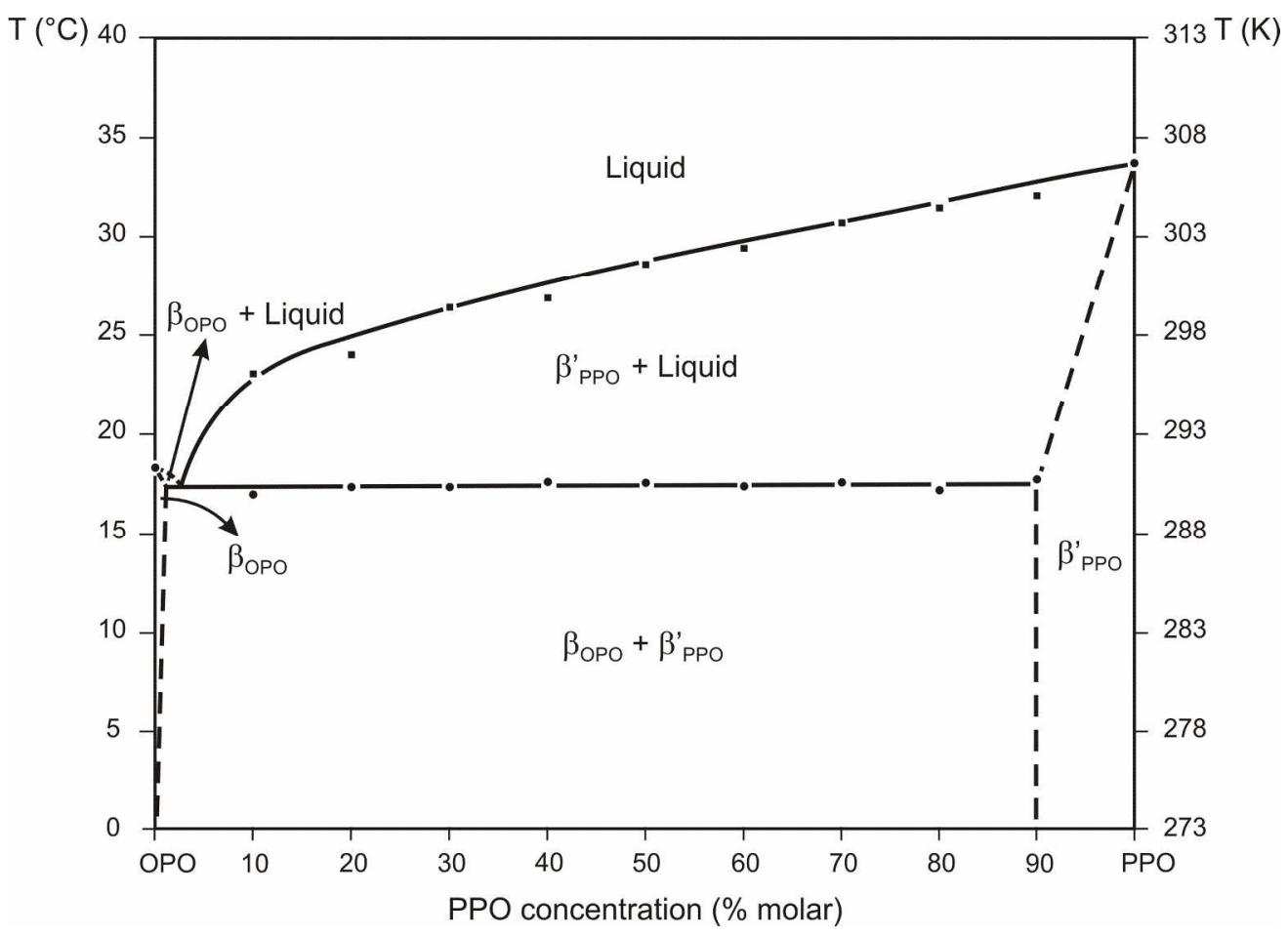

Phase behavior of PPO-OPO mixtures, based on DSC onset and end temperatures ( $\left.T_{\text {end }}-\Delta T_{\text {end }}\right)$. Dashed lines represent solubility domain limits, which were defined by extrapolation, not by precise experimental determination. 


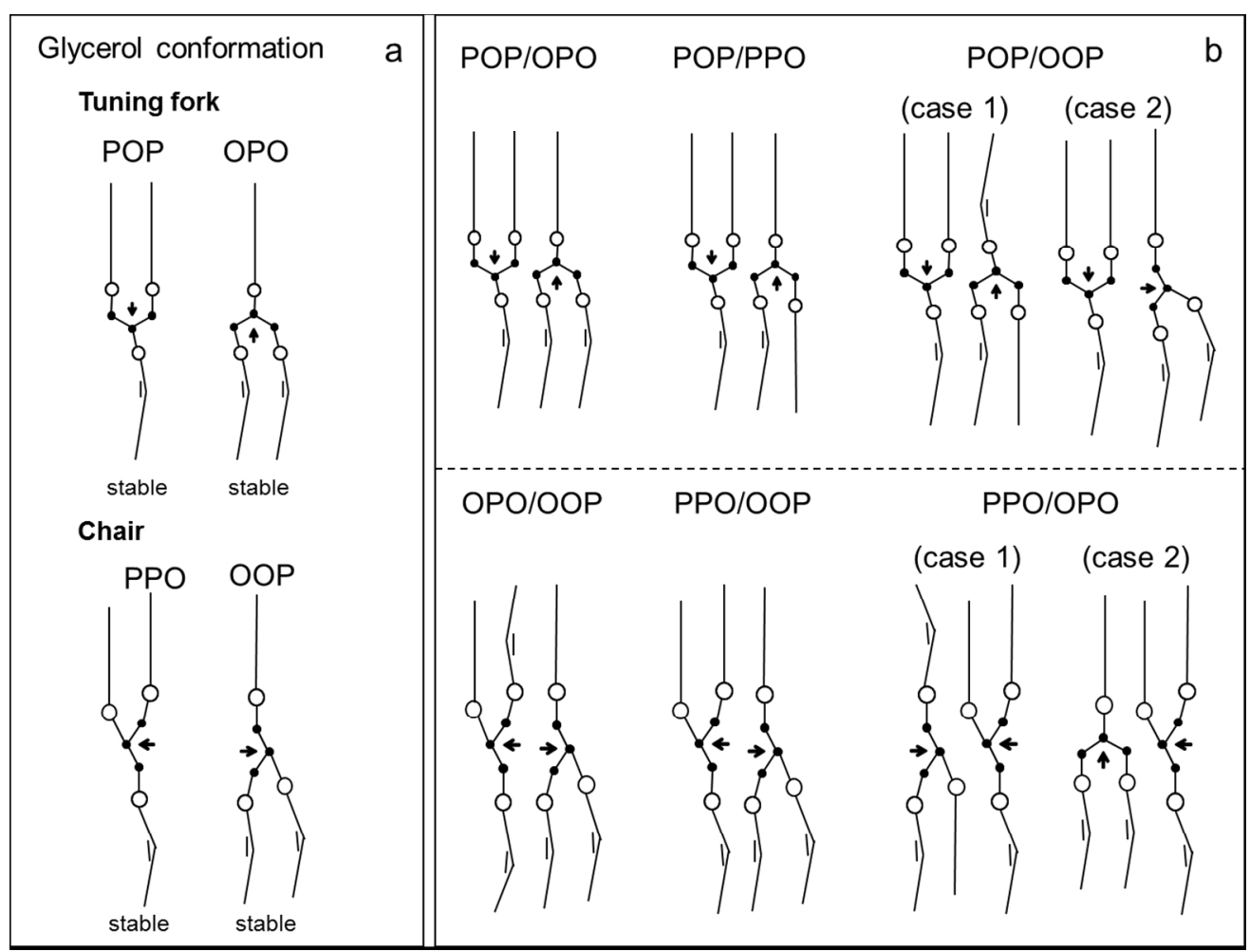

a) Structure models of TAGs containing palmitic and oleic fatty acids (tuning fork and chair conformation). b) Structure models of molecular compounds of TAGs containing palmitic and oleic fatty acids. POP-OPO, POP-PPO and POP-POO cases were reported by Minato et al. ${ }^{20,19}$ and Zhang et al. ${ }^{18}$, respectively. $232 \times 175 \mathrm{~mm}(150 \times 150 \mathrm{DPI})$ 